\title{
A novel redox-sensitive system based on single- walled carbon nanotubes for chemo-photothermal therapy and magnetic resonance imaging
}

This article was published in the following Dove Press journal:

International Journal of Nanomedicine

5 February 2016

Number of times this article has been viewed

\author{
Lin $\mathrm{Hou}^{1,2}$ \\ Xiaomin Yang' \\ Junxiao Ren' \\ Yongchao Wang' \\ Huijuan Zhang' \\ Qianhua Feng' \\ Yuyang Shi' \\ Xiaoning Shan' \\ Yujie Yuan' \\ Zhenzhong Zhang ${ }^{1,2}$ \\ 'School of Pharmaceutical Sciences, \\ Zhengzhou University, Henan \\ Province, Zhengzhou, People's \\ Republic of China; ${ }^{2}$ Collaborative \\ Innovation Center of New Drug \\ Research and Safety Evaluation, \\ Henan Province, Zhengzhou, People's \\ Republic of China
}

Correspondence: Zhenzhong Zhang School of Pharmaceutical Sciences, Zhengzhou University, No 100,

Kexueroad, Zhengzhou 45000I, People's Republic of China

$\mathrm{Tel}+8637167781910$

Fax +86 37I 67781908

Email zhangzz_pharm@163.com
Abstract: Recently, nanomaterials with multiple functions, such as drug carrier, magnetic resonance imaging (MRI) and optical imaging, and photothermal therapy, have become more and more popular in cancer research. In this work, a novel redox-sensitive system constructed from hyaluronic acid (HA), single-walled carbon nanotubes (SWCNTs), doxorubicin (DOX), and gadolinium $(\mathrm{Gd})$ was successfully developed. Herein, HA-modified SWCNTs (SWCNTs-HA) was first synthesized, and then DOX was conjugated with HA by disulfide bond (SWCNTs-HAss-DOX). Finally, MRI contrast agents, $\mathrm{Gd}^{3+}$-ion loading occurred through the sidewall defects of SWCNTs, whose cytotoxicity could be sequestered within the SWCNTs. In vitro release of DOX showed that this system accomplished much faster drug release under reducing condition. Confocal microscopy analysis confirmed that Gd/SWCNTs-HA-ss-DOX were capable of simultaneously delivering DOX and SWCNTs into Michigan Cancer Foundation-7 cells via HA receptor-mediated endocytosis followed by rapid transport of cargoes into the cytosol. Enhanced cytotoxicity of Gd/SWCNTs-HA-ss-DOX further proved that the sensitive system was more potent for intracellular drug delivery as compared with the insensitive control. Meanwhile, tumor cell killing potency was improved when Gd/SWCNTs-HA-ss-DOX were combined with near-infrared irradiation, with $\mathrm{IC}_{50}$ of $0.61 \mu \mathrm{g} / \mathrm{mL}$ at 48 hours. In vivo investigation demonstrated that Gd/SWCNTs-HA-ss-DOX could effectively accumulate in tumor sites and possessed the greatest synergistic antitumor efficacy, especially under the $808 \mathrm{~nm}$ laser irradiation. More importantly, this system could be used as a contrast agent for MRI to identify the location and extent of tumor tissues. These results suggested that Gd/SWCNTs-HA-ss-DOX might be a promising system for targeting chemo-photothermal therapy and MRI diagnosis in future clinical anticancer applications.

Keywords: redox-responsive, tumor targeting, cancer diagnosis, synergistic effect, multifunctional

\section{Introduction}

Theranostics is a newly emerging concept which involves simultaneous execution of therapeutic and diagnostic approaches for personalized medicine. ${ }^{1}$ It allows real-time evaluation of therapeutic effects in vivo, which is critical for medical intervention through noninvasive methods. ${ }^{2}$ In this respect, nanocarriers which can simultaneously perform multiple functions, such as tumor targeting, imaging ultrasensitivity, and drug delivery, have become more and more popular in the domain of cancer therapy. So far, cancer researchers have been actively exploring various tumor-targeting nanoparticles made of lipid-based micelles, natural/synthetic polymeric particles, and inorganic particles for cancer theragnosis. ${ }^{3,4}$

Magnetic resonance imaging (MRI), which produces high-resolution three-dimensional maps delineating morphological features of the specimen and lack of ionizing radiation, 
is one of the best strategies used in clinic to diagnose cancer. ${ }^{5}$ However, there remains the issue of insufficient contrast between the healthy and diseased tissues in a correspondingly low apparent MRI sensitivity for identifying the associated abnormalities. ${ }^{6}$ Consequently, contrast agents (CAs) have been used to overcome this limitation. Among different kinds of CAs, gadolinium (Gd)-based CAs are employed in the overwhelming majority of cases. But the aquated $\mathrm{Gd}^{3+}$ ion is toxic and Gd-chelates suffer from nonspecific biodistribution and fast elimination. In recent work, $\mathrm{Gd}^{3+}$-based carbon nanotubes with unique nanoscalar properties and superior performance as MRI CAs have shown promise for molecular imaging and other advanced applications. ${ }^{7,8}$

Single-walled carbon nanotubes (SWCNTs) are one of the most promising delivery vehicles for cancer diagnosis and therapy due to their significant advantages over the spherical nanoparticles, including high drug-carrying capacities, prolonged circulating time, and remarkable cell membrane penetrability. ${ }^{9}$ In particular, SWCNTs have strong optical absorption in the near-infrared (NIR) region and are prospective photothermal therapy agents for in vivo tumor destruction. ${ }^{10}$ Sitharaman et $\mathrm{al}^{7}$ reported the nanoscale loading of aquated $\mathrm{Gd}^{3+}{ }_{n}$-ion clusters within ultrashort SWCNTs, and found that the MRI efficacies of these species were 40-90 times larger than any $\mathrm{Gd}^{3+}$-based CAs in current clinical use. Current developments in synthesis of new SWCNTs-based carriers were coated with suitable materials and conjugated with tumor-specific moieties for improving biocompatibility and targeting capabilities. ${ }^{11}$

In various materials for carrier modification, hyaluronic acid (HA) has been extensively investigated for design of these advanced biomaterials, owing to its biocompatibility and biodegradability. ${ }^{12}$ Importantly, HA can specifically bind to cancer cells that overexpress CD $44,{ }^{13}$ resulting in the belief that CD44-HA interaction may provide a new approach to the targeted diagnosis and treatment of specific cancers. ${ }^{14}$ Respecting these advantages of HA, we synthesized HAmodified SWCNTs (SWCNTs-HA) to further prepare the $\mathrm{Gd}^{3+}$-based CAs in this work.

Moreover, in order to achieve the pairing of a therapeutic agent with a diagnostic technique, anticancer drugs would be also loaded to the nanocarriers. Complexation of drug molecules to nanocarriers, such as SWCNTs, may be accomplished via physical association or by covalent conjugation. ${ }^{15}$ Physical association of drug with the vehicle has the potential disadvantage of rapid and poorly controlled release due to the equilibration of drug with the external matrix. ${ }^{16}$ In contrast, generation of a covalent drug-carrier conjugate has the potential to facilitate controlled drug release via environmental-responsive bond. Disulfide bonds, which are stable in the mildly oxidizing extracellular milieu, may be prone to rapid cleavage through thiol-disulfide exchange reactions with intracellular reducing molecules, especially with glutathione (GSH). ${ }^{17}$ The high redox potential in the cytosol and cell nuclei that contain 100-1,000 times higher concentration of reducing GSH tripeptide than body fluids including blood and extracellular milieu (0.5-10 mM vs 2-20 $\mu \mathrm{M}$ GSH) have been exploited for active intracellular release of various drugs. ${ }^{18,19}$ It should further be noted that tumor tissues are highly hypoxic with at least fourfold higher GSH levels relative to normal tissues. ${ }^{20}$ This significant difference in GSH level has rendered GSH-responsive nanovehicles most appealing for tumor targeting drug delivery. In this study, SWCNTs-HA have been developed into an active targeting multifunctional theragnostic vehicle to carry both anticancer drug, doxorubicin (DOX), and MRI CAs (Figure 1). Furthermore, NIR photothermal properties of SWCNTs might show combined and synergistic treatment effect. The synthesized DOX conjugated with HA by redox-sensitive disulfide bond (SWCNTs-HA-ss-DOX) was analyzed by Fourier transform infrared spectroscopy (FTIR), ultraviolet-visible spectroscopy, and proton nuclear magnetic resonance $\left({ }^{1} \mathrm{HNMR}\right)$. The properties and performance were characterized by transmission electron microscopy (TEM), scanning electron microscopy, flow cytometry, and confocal microscopic analyses. Finally, the theragnostic potential of the $\mathrm{Gd}^{3+}$-ion-loaded SWCNTs-HA-ss-DOX was evaluated by their tumor specificity, targeted delivery of the drug, and in vivo monitoring of the therapeutic responses, simultaneously.

\section{Materials and methods Materials}

All animal experiments were performed under a protocol approved by Henan laboratory animal center. All animalhandling procedures were performed according to the animal care guidelines. SWCNTs (weight: $2 \mathrm{~g}$, optical density: 1-2 nm, length: $1-3 \mu \mathrm{m}$, purity: $>90 \mathrm{wt} \%$, ash: $<1.5 \mathrm{wt} \%$, specific surface area: $>380 \mathrm{~m}^{2} / \mathrm{g}$, and electrical conductivity: $>102 \mathrm{~s} / \mathrm{cm}$ ) were purchased from Chengdu Organic Chemicals Co., Ltd. (Sichuan, People's Republic of China). HA (molecular weight $12 \mathrm{kDa}$ ) was purchased from Bloomage Freda Biopharm Co., Ltd. (Shandong, People's Republic of China). 1-Ethyl-3 (3-dimethylaminopropyl) carbodiimide, $N$-hydroxysuccinimide, dithiothreitol, 3,3'-dithiodipropionic acid and GSH were purchased from Aladdin Reagent Database Inc. (Shanghai, People's Republic of China). 


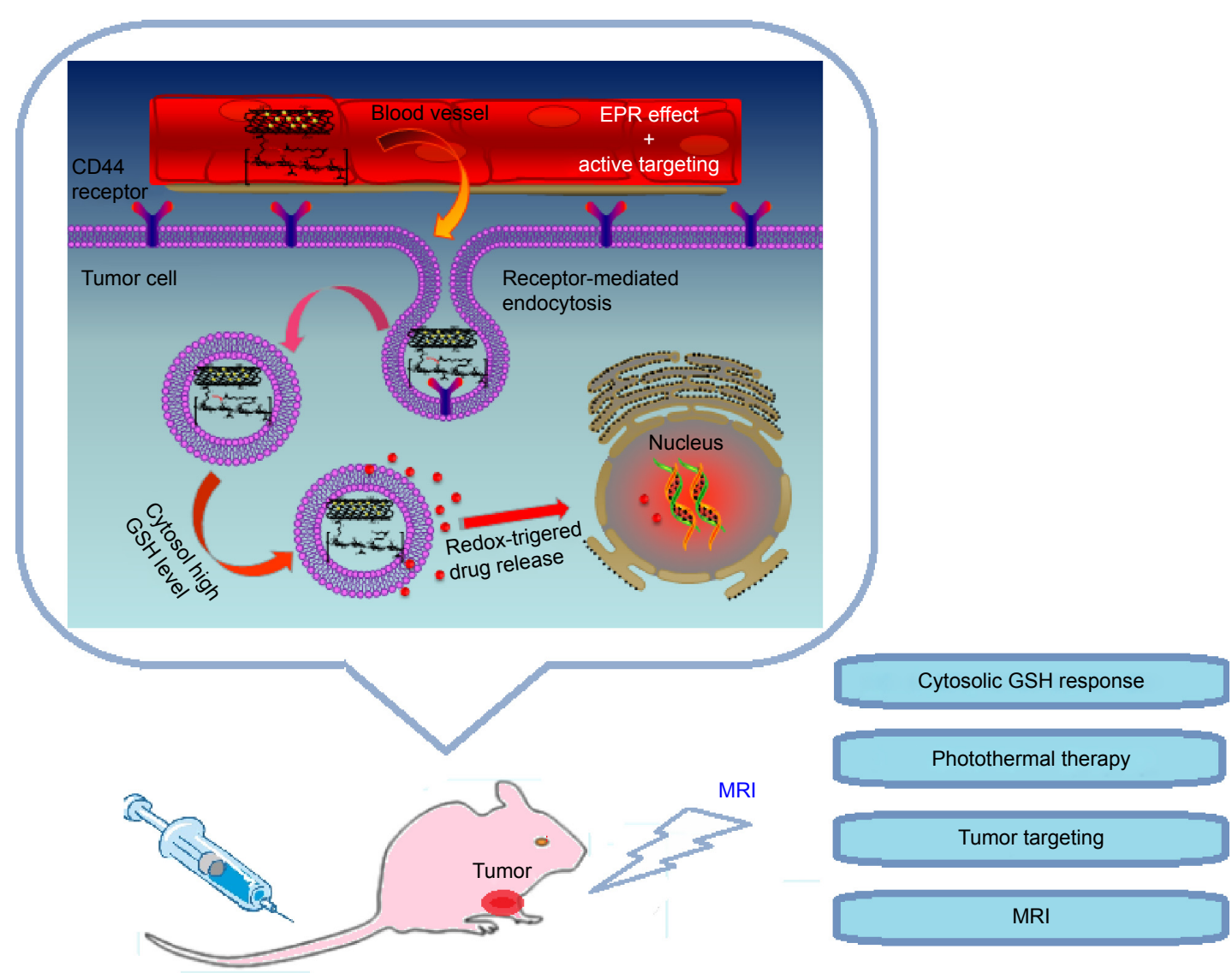

Figure I Schematic illustration of redox-sensitive Gd/SWCNTs-HA-ss-DOX for HA-mediated endocytosis, GSH triggered intracellular drug release and its application. Abbreviations: DOX, doxorubicin; EPR, enhanced permeability and retention; Gd, gadolinium; GSH, glutathione; HA, hyaluronic acid; MRI, magnetic resonance imaging; SWCNTs, single-walled carbon nanotubes.

Hydroxylammonium chloride, 3-mercaptopropionic acid, and poloxamer 188 were purchased from Tianjin Fengchuan Chemical Reagent Technologies Co., Ltd. (Tianjin, People's Republic of China). DOX was purchased from Dalian Meilun Biotech Co., Ltd. (Liaoning, People's Republic of China). L- $\alpha-$ Phosphatidylcholine was purchased from Beijing Solarbio Co., Ltd. (Beijing, People's Republic of China). Cell cycle kit and Apoptosis Assays kit were purchased from Beyotime Institute of Biotechnology Co., Ltd. (Shanghai, People's Republic of China). IR-783 was purchased from Beijing Fanbo Biochemicals Co., Ltd. (Beijing, People's Republic of China).

\section{Synthesis and characterization of SWCNTs- HA-ss-DOX and SWCNTs-HA-DOX Synthesis of SWCNTs-HA-ss-DOX and SWCNTs- HA-DOX}

The SWCNTs-HA was synthesized as described in our previous report. ${ }^{21}$

SWCNTs-HA-ss-COOH was synthesized by a coupling reaction. In a typical procedure, 3,3'-dithiodipropionic acid (1 g, $5 \mathrm{mM}$ ), 1-ethyl-3 (3-dimethylaminopropyl) carbodiimide
( $1 \mathrm{~g}, 5.56 \mathrm{mM})$, and $N$-hydroxysuccinimide $(0.5 \mathrm{~g}, 4.35 \mathrm{mM})$ were dissolved in $20 \mathrm{~mL}$ of anhydrous formamide. SWCNTs-HA $(0.2 \mathrm{~g}, 3.5 \mu \mathrm{M})$ were then added to the solution, and the mixture was stirred at room temperature for 4 hours. After reaction completion, the mixture was treated in excess acetone and dialyzed against distilled water for 2 days using a dialysis membrane (molecular weight cutoffs [MWCO] 12 $\mathrm{kDa})$. The resulting solution was lyophilized.

DOX (50 mg, $0.086 \mathrm{mM}$ ) and SWCNTs-HA-ss-COOH $(127.3 \mathrm{mg}, 2.0 \mu \mathrm{M})$ were dissolved in $1 \mathrm{~mL}$ of phosphatebuffered saline (PBS) solution and $0.5 \mathrm{~mL}$ of dimethyl sulfoxide, respectively. After reaction at room temperature for 2 hours, hydroxylamine hydrochloride $(526.5 \mathrm{mg}, 7.58 \mathrm{mM})$ was added to the mixture as above, followed by stirring for another 2 hours. The resulting solution was dialyzed against the excess amount of water for 2 days. After being freezedried, the final products were obtained as a dark red powder and stored at $4{ }^{\circ} \mathrm{C}$ until further use.

The synthesis procedure of SWCNTs-HA-DOX was similar to that of SWCNTs-HA-ss-DOX, but 3,3'-dithiodipropionic acid was replaced with adipic acid. 


\section{Characterization}

FTIR spectra were recorded on a Nicolet iS10 spectrometer (Thermo Fisher Scientific, Waltham, MA, USA) and ${ }^{1} \mathrm{HNMR}$ spectrum was obtained on a Bruker DPX 300 spectrometer. Moreover, the morphology of SWCNTs, SWCNTs-HA, SWCNTs-HA-ss-DOX, and SWCNTs-HA-DOX were characterized by TEM and hyperspectral microscopy, respectively. The DOX content in the SWCNTs-HA-ss-DOX was determined by fluorescence measurement. Briefly, $0.25 \mathrm{mg} / \mathrm{mL}$ of SWCNTs-HA-ss-DOX solution was mixed with $10 \mathrm{mM}$ of dithiothreitol in the nitrogen. After stirring for 4 hours, the resulting solution was ultrafiltered and detected using fluorospectrophotometer with the wavelength of excitation at $480 \mathrm{~nm}$ and emission at $557 \mathrm{~nm}$. For SWCNTs-HADOX, the content of DOX was also estimated by fluorescence measurement based on a standard curve generated with known concentrations of DOX in ethanol.

\section{Preparation and characterization of $\mathrm{Gd}^{3+}$-ion-loaded SWCNTs-HA-ss-DOX}

$\mathrm{Gd}^{3+}$-ion loading onto SWCNTs-HA-ss-DOX (Gd/SWCNTsHA-ss-DOX) was done by simply mixing $9 \mathrm{mg} / \mathrm{mL}$ of $\mathrm{GdCl}_{3}$ with SWCNTs-HA-ss-DOX solution $(\sim 1 \mathrm{mg} / \mathrm{mL})$ for 30 minutes at room temperature. The resulting complexes were centrifuged at 10,000 rpm for 10 minutes, the supernatant was removed, and then resuspended by pluronic $\mathrm{F} 68$ plus phospholipid $(1: 2, w / w)$ solution. Finally, the solution was dialyzed against an excess amount of distilled water with a dialysis bag (MWCO $12 \mathrm{kDa}$ ) for 2 days. The amount of $\mathrm{GdCl}_{3}$ in the nanocarriers was measured by inductive-coupled plasmaoptical emission spectrometry. As control, $\mathrm{Gd}^{3+}$-ion-loaded SWCNTs-HA-DOX was also prepared in the same method.

\section{In vitro drug release}

The in vitro DOX release was conducted in PBS $(\mathrm{pH} 7.4$, $0.01 \mathrm{M}$ ) with different GSH levels $(0$ and $10 \mu \mathrm{M}$, and 10 and $20 \mathrm{mM}$ ) and gently shaken at $37^{\circ} \mathrm{C}$ in a water bath at $100 \mathrm{rpm}$. One mg Gd/SWCNTs-HA-ss-DOX freeze-dried powder was dispersed in $1 \mathrm{~mL}$ PBS in a dialysis bag (MWCO 3,500). At predetermined time intervals, $2 \mathrm{~mL}$ release media was withdrawn and replaced with $2 \mathrm{~mL}$ of the corresponding fresh medium. The amount of DOX release was determined by fluorescence measurement. The drug release from $\mathrm{Gd} /$ SWCNTs-HA-DOX in PBS with or without GSH was also measured as control.

\section{In vitro cell culture and cellular uptake study}

The Michigan Cancer Foundation-7 (MCF-7) human breast cancer cell line was obtained from the Chinese Academy of
Sciences Cell Bank (Catalog No TCHu 74), and cells were maintained in Gibco RPMI 1640 supplemented with 10\% bovine serum in a humidified atmosphere of $95 \%$ air and $5 \% \mathrm{CO}_{2}$ at $37^{\circ} \mathrm{C}$ in a humidified incubator.

To evaluate the intracellular uptake capacities of nanotube formulation by fluorescent microscope, fluorescence probe fluorescein isothiocyanate (FITC) was encapsulated in SWCNTs-HA, SWCNTs-HA-ss-DOX, Gd/SWCNTsHA-ss-DOX, and Gd/SWCNTs-HA-DOX by mixing FITC solution with them for 12 hours at room temperature and then repeated washing to remove unbound FITC.

For fluorescent microscope studies, MCF-7 cells were seeded on culture slides at a density of $5.0 \times 10^{5}$ cells/well (surface area of $1.7 \mathrm{~cm}^{2} /$ well, 4 chamber slides) and incubated for 24 hours at $37^{\circ} \mathrm{C}$. FITC, alone or entrapped in the nanotubes, was added and incubated for 1, 2, 4, and 6 hours, respectively. The next treatment procedure was just the same as our previous investigations. ${ }^{21}$ On the other hand, DOX, Gd/SWCNTs-HAss-DOX, and Gd/SWCNTs-HA-DOX were also studied as above, except for observing the fluorescence of DOX.

Moreover, confocal laser scanning microscopy was employed to visualize the intracellular distribution and investigate whether nanotube formulation was specifically taken up through HA receptor-mediated endocytosis. Cells were treated with free HA for 2 hours before the samples were added. At the designated time points ( 1 and 6 hours after incubation), the cells were washed three times with PBS and fixed with $4 \%$ paraformaldehyde for 10 minutes, followed by adding 4',6-diamidino-2-phenylindole to stain the cell nuclei. At last, the cells were observed using Olympus FluoView

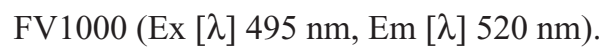

\section{In vitro cytotoxicity assays}

To further verify that enhanced in vitro antitumor activity could be achieved by the redox-sensitive formulation, the cytotoxicity of DOX, SWCNTs-HA-ss-DOX, Gd/SWCNTsHA-ss-DOX, and Gd/SWCNTs-HA-DOX was evaluated in MCF-7 cells. MCF-7 cells were placed into 96-well plates at $5.0 \times 10^{3}$ cells/well and were allowed to adhere prior to addition of varying concentrations of DOX, SWCNTs-HAss-DOX, Gd/SWCNTs-HA-ss-DOX, and Gd/SWCNTs-HADOX (containing 0, 0.039, 0.078, 0.156, 0.313, 0.625, 1.25, 2.5 , and $5 \mu \mathrm{g} / \mathrm{mL}$ of DOX). Then the cells were or were not irradiated with an $808 \mathrm{~nm}$ continuous-wave NIR laser with the power density of $2 \mathrm{~W} / \mathrm{cm}^{2}$ for 3 minutes. After that, the cells were incubated at $37^{\circ} \mathrm{C}$ for further 24,48 , and 72 hours. Sulforhodamine B assay was used to measure the cell viability at the given time intervals. The drug concentration which caused a $50 \%$ inhibition of the control growth rate 
$\left(\mathrm{IC}_{50}\right)$ was calculated by nonlinear regression analysis using the equation for a sigmoid plot.

\section{Cell cycle and apoptosis assays}

To confirm the therapeutic effect of these formulations, we performed the cell apoptosis and cell cycle assays by flow cytometry. First, MCF-7 cells were seeded in 6-well plates at a density of $1 \times 10^{5}$ cells/well and incubated at $37^{\circ} \mathrm{C}$ for 24 hours. Then the cells were treated with DOX, SWCNTs-HA-ss-DOX, Gd/ SWCNTs-HA-ss-DOX, and Gd/SWCNTs-HA-DOX containing $5 \mu \mathrm{g} / \mathrm{mL}$ equivalent DOX to incubate for future 24 hours. For the hyperthermia groups, these samples were treated with $808 \mathrm{~nm}$ laser for 1.0 minutes after incubation for 2 hours. The rate of apoptosis was measured by using Annexin V-FITC Apoptosis Detection Kit (Sigma-Aldrich Co. LLC, St Louis, MO, USA) in accordance with the manufacturer's protocol.

\section{In vivo $M R I$}

Approximately $1 \times 10^{6} \mathrm{~S} 180$ cells were inoculated subcutaneously in the armpit region of BALB/c male mice ( 7 weeks old, 20-25 g).

When the tumors reached about $500 \mathrm{~mm}^{3}$, solutions of GdDTPA, Gd/SWCNTs-COOH, and Gd/SWCNTs-HA-ss-DOX $\left(\mathrm{Gd}^{3+}, 0.05 \mathrm{mmol} / \mathrm{kg}\right)$ were intravenously injected via the tail veins. $T_{1}$-weighted images of the mice were acquired using a Litz coil (diameter $100 \mathrm{~mm}$, length $85 \mathrm{~mm}$, Doty Scientific Inc., Columbia, SC, USA) on a $1.5 \mathrm{~T}$ clinical MRI scanner (Signa Excite; GE Healthcare, Milwaukee, WI, USA). The following parameters were adopted: spin-echo method, repetition time $=400 \mathrm{~ms}$, echo time $=11 \mathrm{~ms}$, field of view $=48 \times 48 \mathrm{~mm}$, matrix size $=256 \times 256$, and slice thickness $=2 \mathrm{~mm}$.

\section{In vivo imaging analysis}

For in vivo imaging analysis, NIR dye IR-783 was loaded into SWCNTs-COOH and SWCNTs-HA-ss-DOX with the similar drug-loading content $(\sim 0.5 \%)$ according to the protocol above. When the tumor volume grew to $\sim 500 \mathrm{~mm}^{3}$, mice were injected with free IR-783 and IR-783-labeled SWCNTs samples at a dose of $2.5 \mathrm{mg} / \mathrm{kg}$ via the tail vein. NIR fluorescence imaging experiments were performed at $0.5,1,3,6$, and 8 hours postinjection using a Kodak in vivo imaging system FX PRO (Kodak, Rochester, NY, USA) equipped with an excitation band-pass filter at $750 \mathrm{~nm}$ and an emission at $783 \mathrm{~nm}$. Images were analyzed using the Kodak Molecular Imaging Software 5.X.

\section{In vivo antitumor efficacy studies}

In vivo antitumor efficacy of all formulations alone or in combination with the laser irradiation treatment was assessed by measuring daily tumor volume with a sliding caliper using S180-bearing mice and eight female BALB/c mice per group (18-20 g), purchased from Henan Laboratory Animal Center (Zhengzhou, People's Republic of China). When tumors grew to $\sim 150-200 \mathrm{~mm}^{3}$, mice were randomly divided into five groups and received intravenous administration of saline, free DOX, SWCNTs-HA-ss-DOX, Gd/SWCNTs-HA-ss-DOX, and $\mathrm{Gd} / \mathrm{SWCNTs-HA-DOX}$ at $4 \mathrm{mg}$ DOX/kg every 2 days for 10 days.

The tumor size was calculated with the equation volume $=0.5 \times\left(a \times b^{2}\right)$, where $a$ is the largest and $b$ is the smallest diameter. The inhibition ratio (IR) was used as another index of antitumor activity of the formulation tested. At the end of the experiment, the animals were sacrificed and the tumors were weighed. IR was defined as follows:

$$
\text { Inhibition ratio }(\%)=\frac{\mathrm{Wc}-\mathrm{Wt}}{\mathrm{Wc}} \times 100 \% \text {, }
$$

where Wc and Wt stand for the average tumor weight for control group and treatment group, respectively.

For the laser group, the treatment was the same as described above with the exception that after administration, the mice then received NIR irradiation using 808 $\mathrm{nm}$ laser with a power density of $2.5 \mathrm{~W} / \mathrm{cm}^{3}$ toward the tumor region of tumor-bearing mice for 1 minute at distance of $10 \mathrm{~cm}$.

To further evaluate the antitumor effect of the formulation as above on the animals, the tumors were excised for pathology. Moreover, the toxicity of the above regimens was determined by monitoring animal behavior and the (relative) weight loss.

\section{Statistical analysis}

All the data were presented as mean \pm standard deviation from three to ten independent measurements in separate independent experiments and were analyzed using descriptive statistic and single-factor analysis of variance.

\section{Results and discussion Synthesis and characterization of SWCNTs- HA-ss-DOX and SWCNTs-HA-DOX}

In this work, SWCNTs-HA-ss-DOX was stable in bloodstream and was selectively taken up to tumor cells via HA receptor-mediated endocytosis, and then rapid drug release was achieved once internalized into tumor cells, thus improving the antitumor efficacy.

As shown in Figures 2 and 3, the synthesis of SWCNTsHA-ss-DOX was confirmed by FTIR and ${ }^{1} \mathrm{HNMR}$ spectra. 

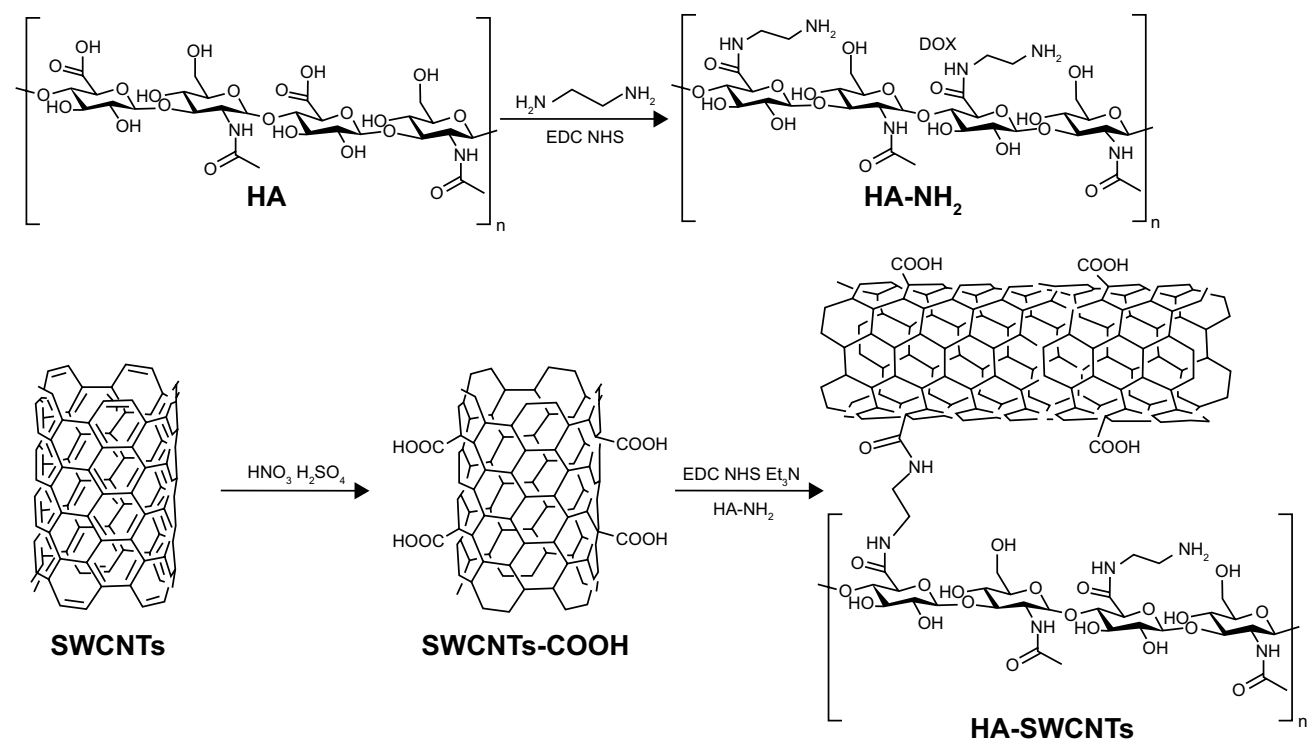

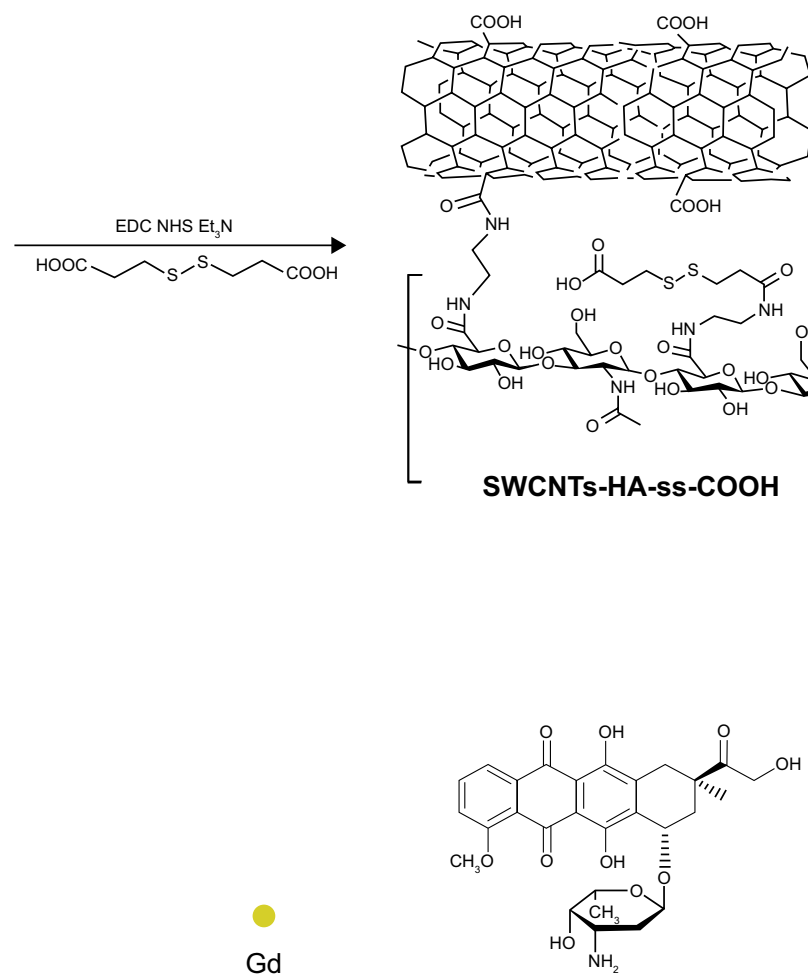

DOX
HA-SWCNTS

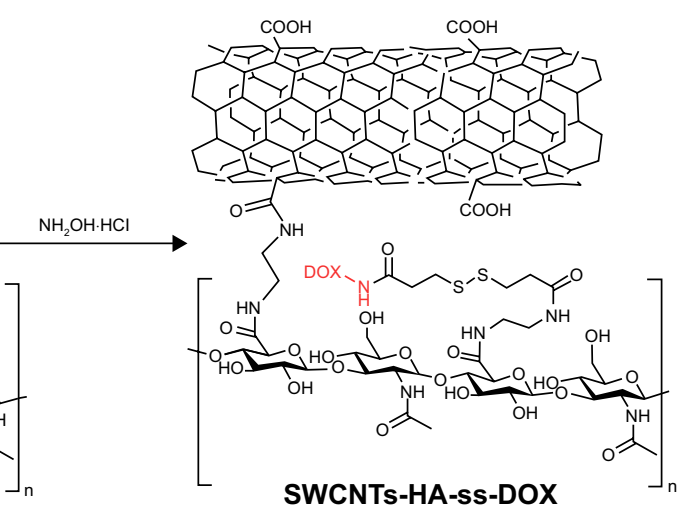

SWCNTs-HA-ss-DOX

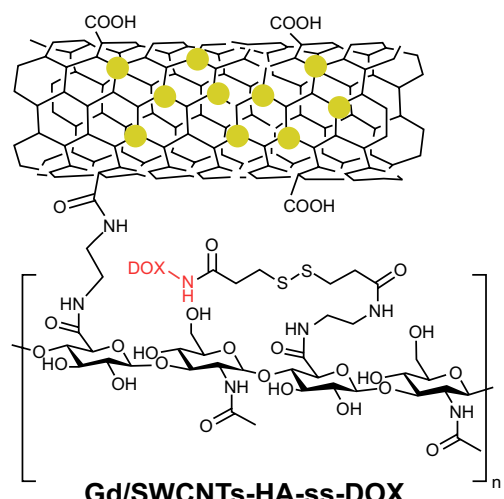

Gd/SWCNTs-HA-ss-DOX

Figure 2 Structures and synthesis of Gd/SWCNTs-HA-ss-DOX

Abbreviations: DOX, doxorubicin; Gd, gadolinium; HA, hyaluronic acid; SWCNTs, single-walled carbon nanotubes.

Compared with HA and SWCNTs, the appearance of characteristic peak around $1,650 \mathrm{~cm}^{-1}$ and the disappearance of the carboxylic group bands at $1,733 \mathrm{~cm}^{-1}$ substantiated the formation of amide linkages. The absorption band at $1,450-1,580$ and $1,600 \mathrm{~cm}^{-1}$ belongs to aromatic ring of DOX. Besides, ${ }^{1} \mathrm{HNMR}$ spectra revealed that the sharp peak at $\delta=2.7 \mathrm{ppm}$ belonged to methylene of 3,3'-dithiodipropionic acid and peaks appeared at $\delta=8.04 \mathrm{ppm}$ indicated the coupling of diacid and free amine of HA. Furthermore, the peaks assigned to both DOX ( $\delta=7.2$ and $7.6 \mathrm{ppm})$ and HA-ss$\mathrm{COOH}(\delta=2.1,3.28-4.75,7.2$, and $7.6 \mathrm{ppm})$ were present in the ${ }^{1} \mathrm{HNMR}$ spectrum. Reduction-insensitive SWCNTs-HADOX was similarly prepared and used as control.

The representative TEM image of SWCNTs and modified SWCNTs are shown in Figure 4A $(a, b)$. Clearly, SWCNTs$\mathrm{COOH}$ appeared to be smooth and without impurities after 


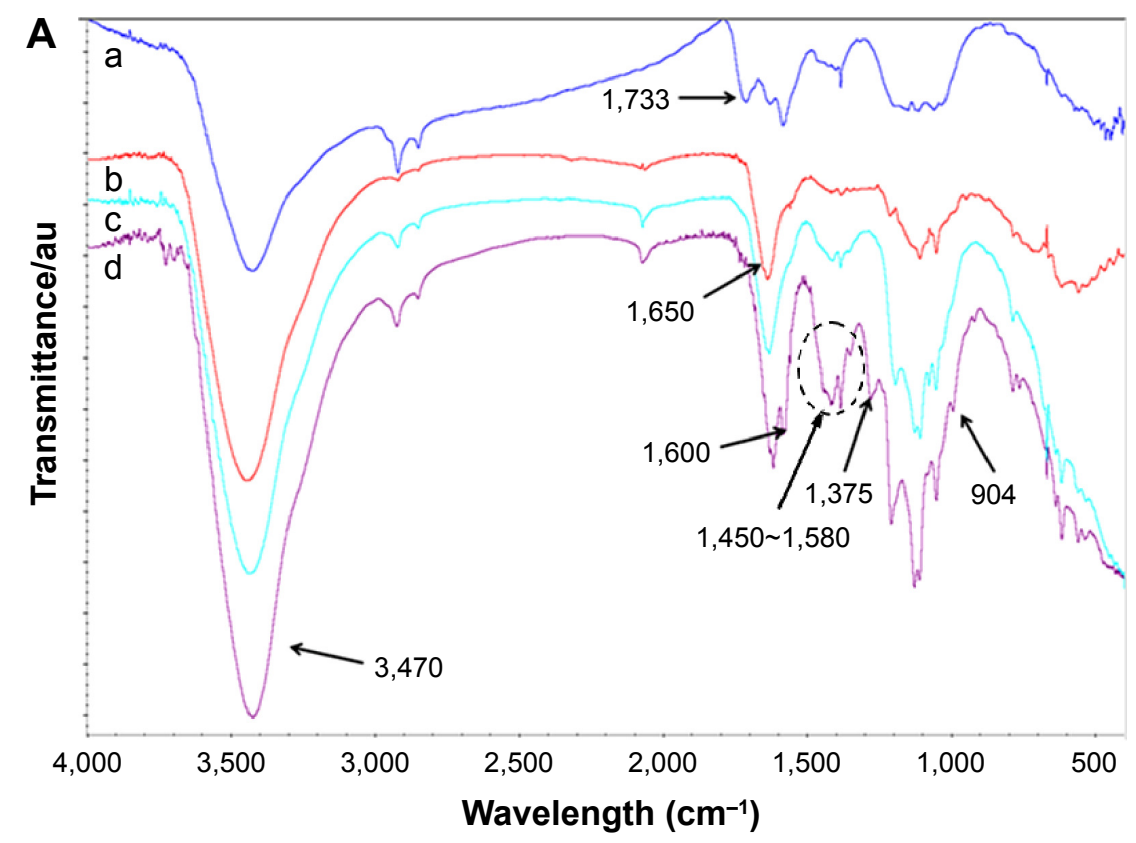

B a

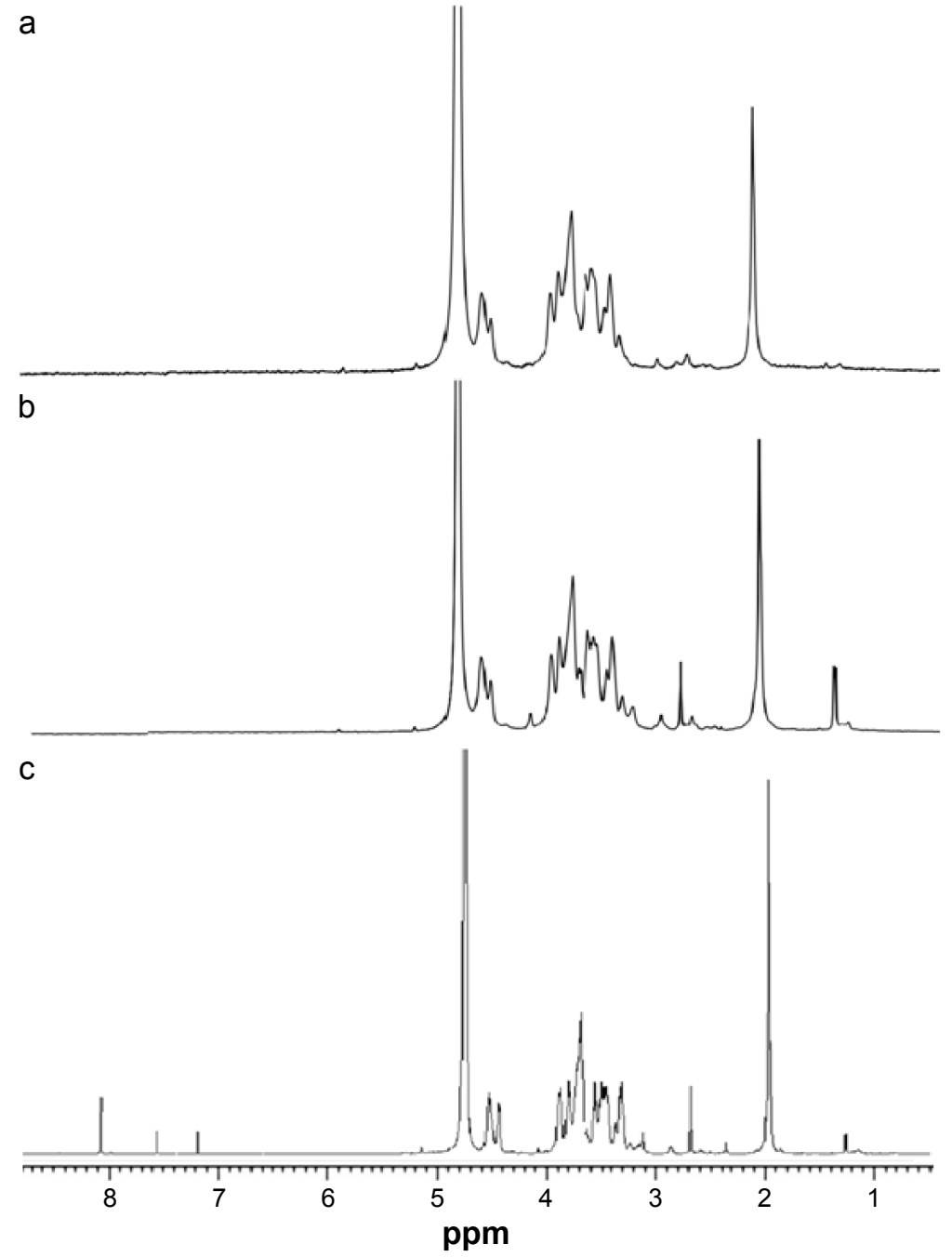

Figure 3 Characterization of different nanotubes.

Notes: (A) FTIR spectrum of a) SWCNTs-COOH, b) SWCNTs-HA, c) SWCNTs-HA-ss- $\mathrm{CH}_{2} \mathrm{CH}_{2}-\mathrm{COOH}$, and d) SWCNTs-HA-ss-DOX. (B) 'HNMR spectra of a) SWCNTs-HA, b) SWCNTs-HA-ss- $\mathrm{CH}_{2} \mathrm{CH}_{2}-\mathrm{COOH}$, and c) SWCNTs-HA-ss-DOX.

Abbreviations: DOX, doxorubicin; FTIR, Fourier transform infrared spectroscopy; Gd, gadolinium; GSH, glutathione; HA, hyaluronic acid; 'HNMR, proton nuclear magnetic resonance; SWCNTs, single-walled carbon nanotubes. 

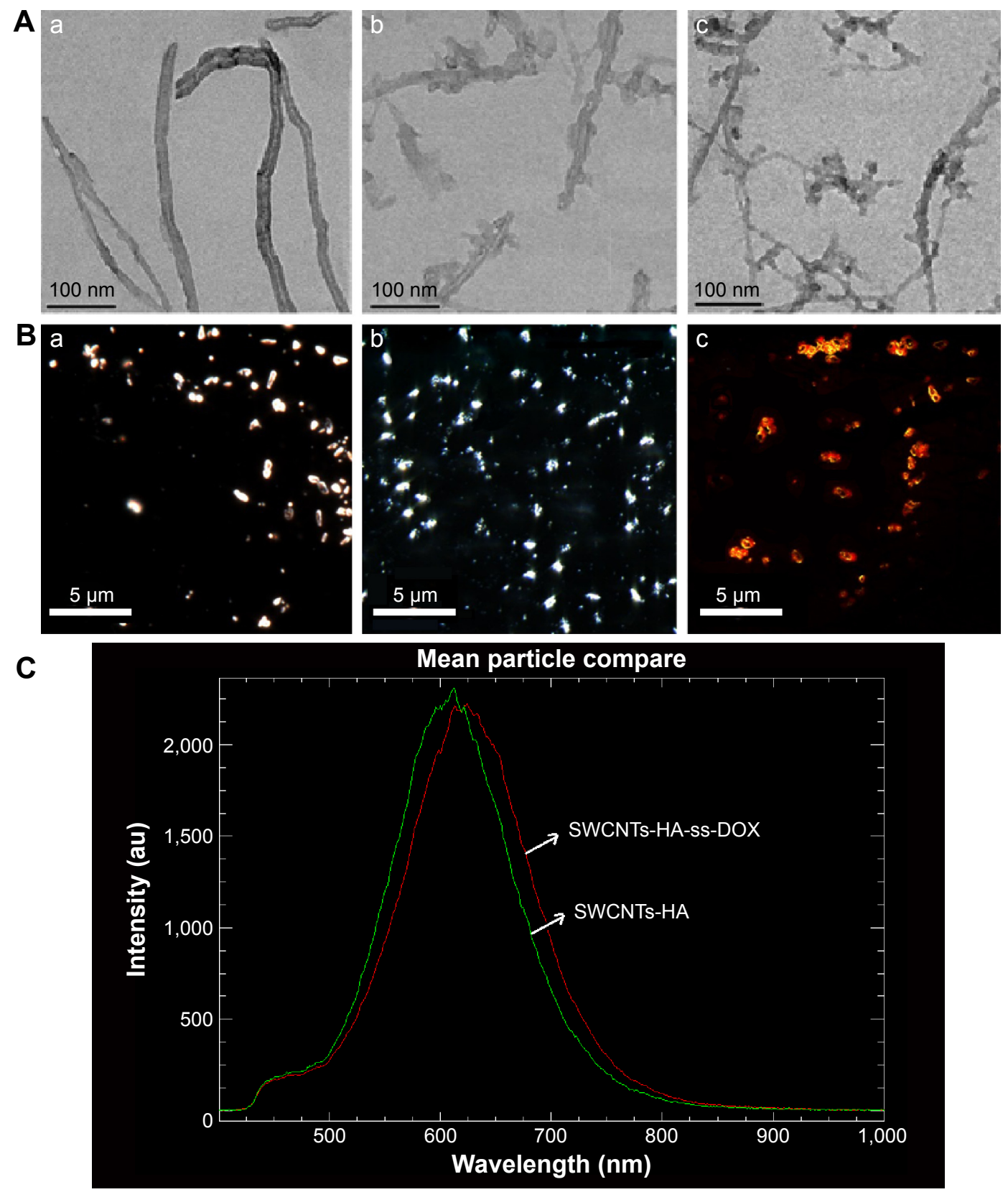

Figure 4 (A) TEM images and (B) hyperspectral images of a) SWCNTs-COOH, b) SWCNTs-HA, and c) SWCNTs-HA-ss-DOX. (C) Mean spectral responses of SWCNTsHA and SWCNTs-HA-ss-DOX.

Abbreviations: DOX, doxorubicin; HA, hyaluronic acid; SWCNTs, single-walled carbon nanotubes.

cutting and cleaning, which indicated that metal particles and amorphous carbon were completely removed. When coated with HA, the polysaccharide chains could be observed on the sidewalls of SWCNTs. Moreover, as shown in Figure 4A (c), when DOX was conjugated with SWCNTs-HA, uniformly coated nanotubes could be obtained. The diameters of the nanotubes were increased and the clarity of the sidewalls was reduced due to screening by both the DOX and the polysaccharides.

In addition, hyperspectral imaging analysis was used as an alternative method to validate the conjugation of HA and DOX with SWCNTs. The morphologies of each sample are shown in Figure 4B. At first, the spectrum of SWCNTs$\mathrm{COOH}$ and SWCNTs-HA was embodied as the white color with the nanotube structure under the light field. By contrast, SWCNTs-HA-ss-DOX showed a red color under the fluorescence channel owing to the fluorescence of DOX. Figure 4C demonstrated the mean spectral responses of SWCNTs-HA and SWCNTs-HA-ss-DOX. The $x$-axis units were in wavelength in nm, and the $y$-axis units were counts of the relative intensity of the reflectance scatter within the spectral data. The peak of SWCNTs-HA-ss-DOX redshifted compared with 
SWCNTs-HA, indicating that the light-scattering properties changed. These data displayed that DOX was successfully conjugated onto the surface of SWCNTs-HA.

The weight percentage (wt\%) of DOX in the SWCNTsHA-ss-DOX was analyzed using fluorospectrophotometer, resulting in $\sim 42.6 \mathrm{wt} \%$.

\section{Preparation and characterization of $\mathrm{Gd}^{3+}$ - ion-loaded SWCNTs-HA-ss-DOX}

The aquated $\mathrm{Gd}^{3+}$ ion is toxic, and therefore for medical use, its toxicity is usually sequestered by chelation with multidentate ligands. ${ }^{22}$ In our study, $\mathrm{Gd}^{3+}$-ion loading may occur through the sidewall defects or end-of-tube openings created by cutting full-length SWCNTs into shortened tubes, whose cytotoxicity could be sequestered within the SWCNTs. Inductive-coupled plasma-optical emission spectrometry analysis showed $26.6 \mu \mathrm{M}$ of $\mathrm{Gd}^{3+}$ adhered to nanodiamonds per milliliter.

\section{In vitro drug release}

As shown in Figure 5, inefficient and slow release of DOX from Gd/SWCNTs-HA-ss-DOX was observed in the absence of GSH. For instance, only $4.4 \%$ of DOX was released in the first 4 hours and $\sim 6.9 \%$ was released within 24 hours. Similar result occurred in the medium with $10 \mu \mathrm{M}$ GSH at $\mathrm{pH}$ 7.4, which mimicked the physicochemical environment in blood circulation. However, in $10 \mathrm{mM} \mathrm{GSH}$, the amount of DOX accumulative release increased with $19.9 \%$ of DOX release in the first 4 hours and near 35.7\% within 24 hours. More importantly, with a higher GSH concentration of $20 \mathrm{mM}$, comparable to intracellular levels reported for GSH in tumor cells, ${ }^{23}$ about $57.7 \%$ of DOX was released in 4 hours and almost $80.2 \%$ was released in 24 hours. It should be further noted that the release of DOX from the control group without redox sensitivity was slow even in the presence of $20 \mathrm{mM}$ $\mathrm{GSH}$, which was similar to the release of DOX from Gd/ SWCNTs-HA-ss-DOX in the absence of GSH. These results indicated that the cleavage of disulfide linkages occurred under the activation of GSH, and Gd/SWCNTs-HA-ss-DOX were highly stable in blood without releasing the drug molecule after intravenously administered, whereas rapid release could be achieved in tumor tissues/cells. ${ }^{24,25}$

\section{In vitro cellular uptake study}

Having confirmed the desired physical and chemical responsiveness of SWCNTs-HA-ss-DOX, we further investigated the cellular uptake of sensitive and nonsensitive nanotubes by fluorescence microscopic analyses. First, to evaluate the tumor cell-specific uptake of HA-modified nanotubes, we tested the internalization of fluorescent probe FITC-loaded nanotubes in MCF-7 cells. As shown in Figure 6A (a-e), almost no fluorescent signals of free FITC were observed until 6 hours. In contrast, SWCNTs-HA, SWCNTs-HA-ss-DOX, Gd/SWCNTs-HA-ss-DOX, and Gd/SWCNTs-HA-DOX

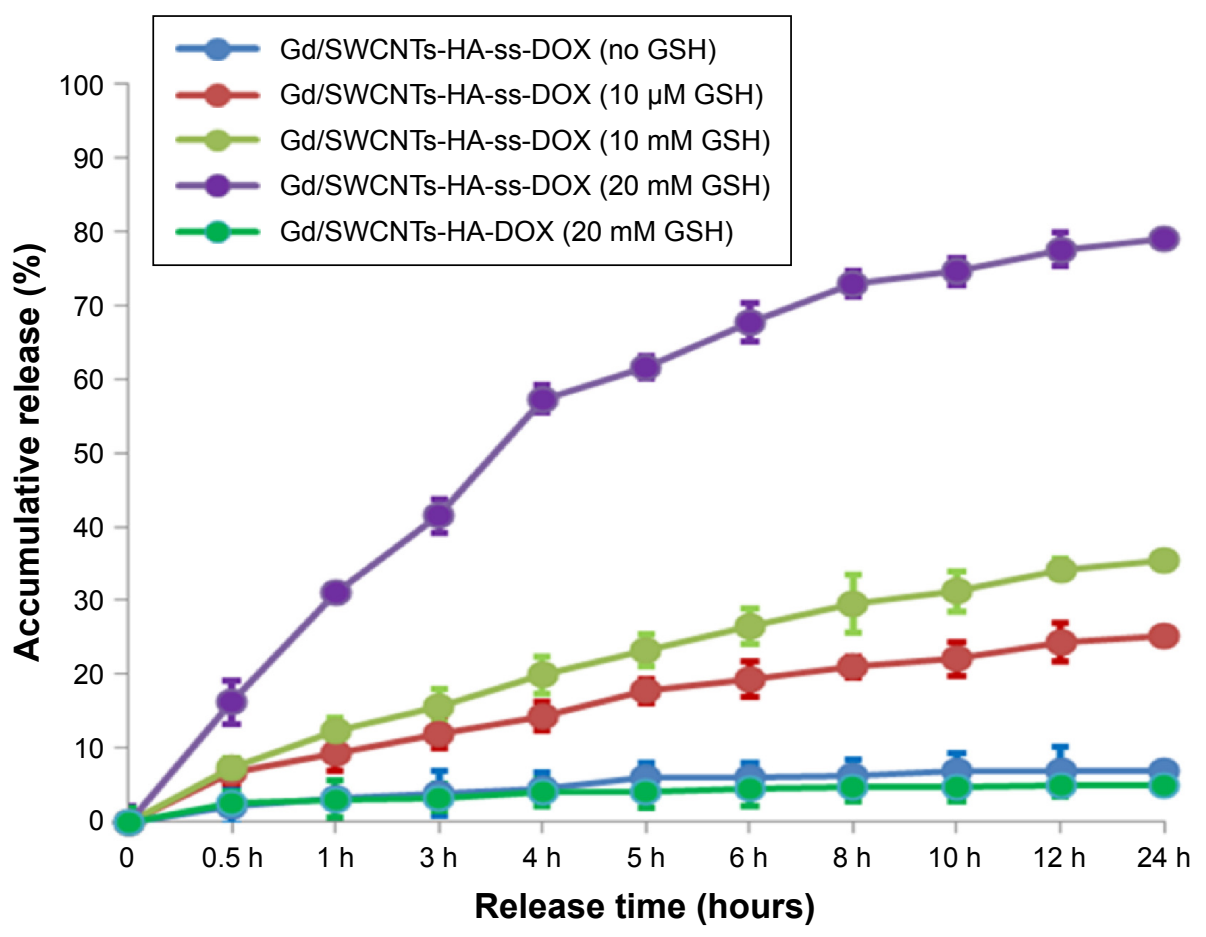

Figure 5 GSH triggered DOX release from Gd/SWCNTs-HA-ss-DOX and Gd/SWCNTs-HA-DOX. The error bars in the graph represent standard deviations (n=3). Abbreviations: DOX, doxorubicin; Gd, gadolinium; GSH, glutathione; HA, hyaluronic acid; SWCNTs, single-walled carbon nanotubes. 
A
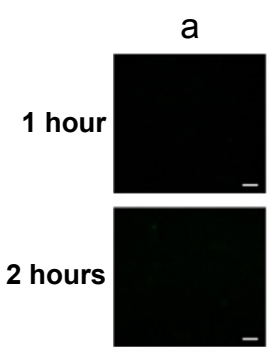

4 hours

6 hours

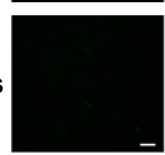

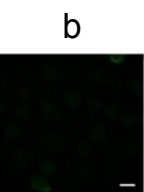
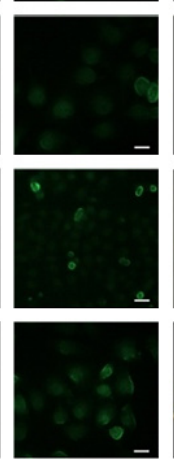
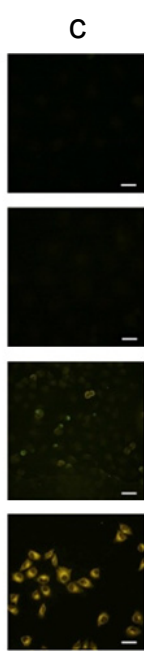
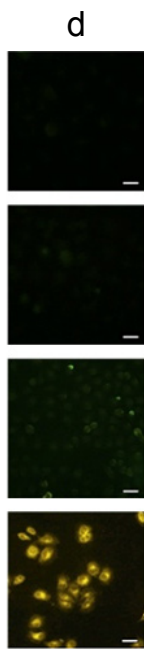
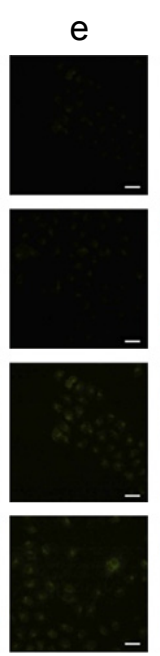
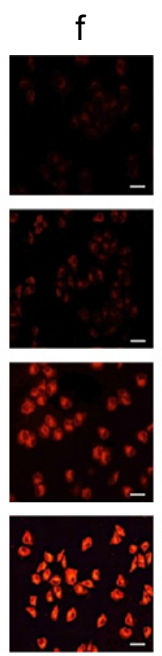
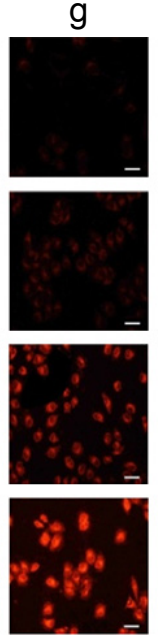

$\mathrm{h}$
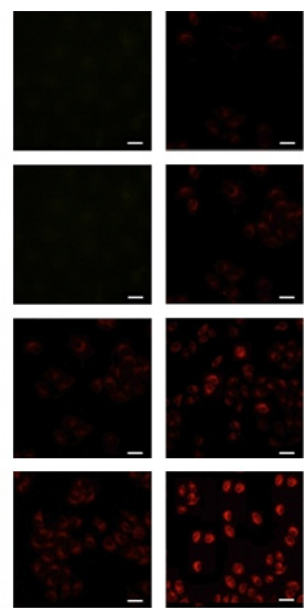

B

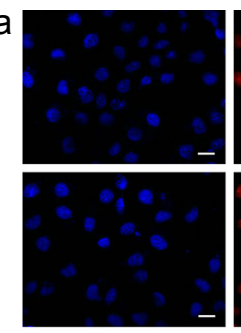

b

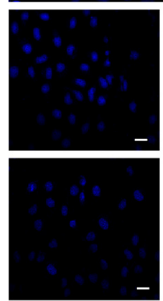

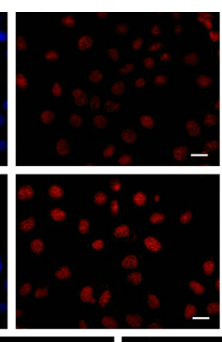

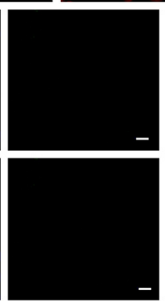

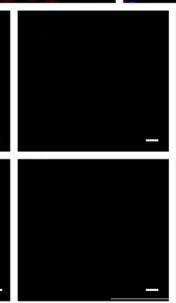
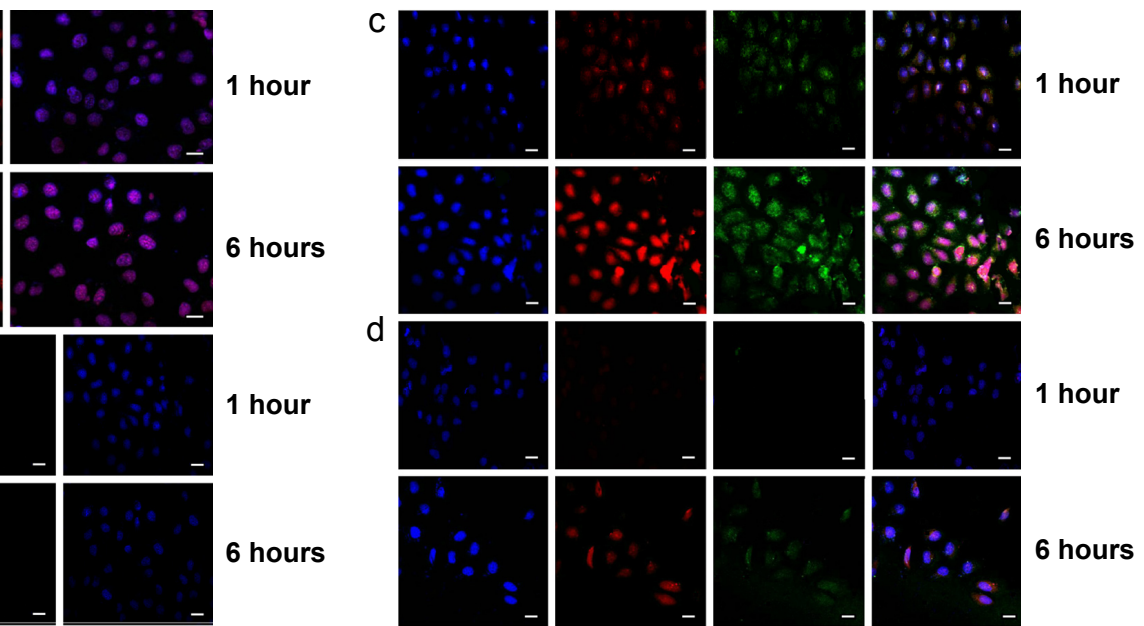

Figure 6 (A) The cellular uptake analyzed by fluorescence microscopy. a) FITC, b) FITC-labeled SWCNTs-HA, c) FITC-labeled SWCNTs-HA-ss-DOX, d) FITC-labeled Gd/SWCNTs-HA-ss-DOX, e) FITC-labeled Gd/SWCNTs-HA-DOX, f) SWCNTs-HA-ss-DOX, g) Gd/SWCNTs-HA-ss-DOX, h) Gd/SWCNTs-HA-DOX, and i) free DOX. (B) The intracellular distribution in MCF-7 cells analyzed by confocal microscopy. a) free DOX, b) free-HA pretreated Gd/SWCNTs-HA-ss-DOX, c) Gd/SWCNTs-HA-ssDOX, and d) Gd/SWCNTs-HA-DOX.

Abbreviations: DOX, doxorubicin; FITC, fluorescein isothiocyanate; Gd, gadolinium; HA, hyaluronic acid; MCF-7, Michigan Cancer Foundation-7; SWCNTs, single-walled carbon nanotubes.

displayed rapid and efficient cellular uptake of FITC, which proved that HA-based nanotubes could specifically bind with and internalized into the MCF-7 cells via CD44 receptor-mediated endocytosis and by the cell-penetrating ability of SWCNTs. In addition, SWCNTs-HA, SWCNTsHA-ss-DOX, Gd/SWCNTs-HA-ss-DOX, and Gd/SWCNTsHA-DOX demonstrated the time-dependent cellular uptake profile and their fluorescent intensities in MCF-7 cells were similar, indicating that loading $\mathrm{Gd}^{3+}$-ion and introducing disulfide conjugated DOX did not affect the cellular uptake behaviors of nanotubes.

On the other hand, the fluorescence signals of DOX in MCF-7 cells were also tracked. Figure 6A (f-i) demonstrated that the redox-sensitive SWCNTs-HA-ss-DOX and Gd/SWCNTs-HA-ss-DOX, exhibiting similar fluorescence intensity during 6 hours of observation, could enter cells much easier compared with free DOX. It suggested that the prepared conjugate could be uptaken by MCF-7 cells more easily and DOX could be released from the vehicle owing to the breakage of disulfide linker via enzymolysis as our design, resulting in cytotoxicity. ${ }^{26}$ Besides, $\mathrm{Gd}^{3+}$-ion attached to the SWCNTs did not prevent the internalization and drug release of the formulation. By contrast, weaker DOX fluorescence was observed for MCF-7 cells incubated for 4 hours with $\mathrm{Gd} /$ SWCNTs-HA-DOX (reduction-insensitive control) under the same conditions. This different intracellular behavior was most likely due to fast cleavage of disulfide bonds in response to the intracellular level of reducing potential.

Then, confocal laser scanning microscopy was employed to follow the redox-induced DOX release and intracellular 
drug distribution in MCF-7 cells (Figure 6B). Notably, the intensity of DOX or FITC fluorescence apparently increased with extended incubation (1 hour and 6 hours). After 1 hour, we found that both DOX and Gd/SWCNTs-HA-ss-DOX (red) were co-localized with the cell nuclei (blue), and showed the pink fluorescence in the merged images. However, DOX carried by $\mathrm{Gd} / \mathrm{SWCNTs-HA-DOX}$ was rarely observed in the nucleus. It has been reported that particles were too large to enter the nuclei pores, ${ }^{27}$ and thus the red fluorescence located in nucleus must be the drug released from nanotubes. As we discussed above, since Gd/SWCNTs-HA-ss-DOX possessed redox sensitivity and experienced cleavage of disulfide linkages in cytoplasm, it could stimulate the intracellular drug release and promote the distribution of cargo into cytoplasm and then the nuclei. Nevertheless, Gd/SWCNTs-HA-DOX, which was stable inside the cancer cell, could not release the conjugated drug and therefore no fluorescence was observed in nucleus. Moreover, the green-color zones of FITC for Gd/SWCNTs-HA-ss-DOX and Gd/SWCNTs-HA-DOX were both found to localize in the cytoplasm, which further indicated that HA-modified nanotubes could be uptaken by the cancer cells and only the released DOX could enter the nucleus.

To further verify the function of HA in facilitating the cellular internalization of nanotubes via interaction with CD44 receptors, MCF-7 cells were incubated with free HA polysaccharide for 2 hours prior to the incubation with $\mathrm{Gd}$ / SWCNTs-HA-ss-DOX and Gd/SWCNTs-HA-DOX. It was found that the fluorescence intensity was significantly inferior to that of untreated one, which demonstrated HA-dependent specific endocytic pathway of nanotubes despite the partial chemical modification of HA. ${ }^{28}$ Taken together, these results displayed that redox-sensitive nanotubes could provide an effective approach for rapid transport of cargo into the cytosol, where the cargo could exert its function.

\section{In vitro cytotoxicity assays}

In general, effective cellular uptake leads to strong cytotoxicity. As shown in Figure 7, higher drug concentration and longer incubation time would cause lower cell viability,
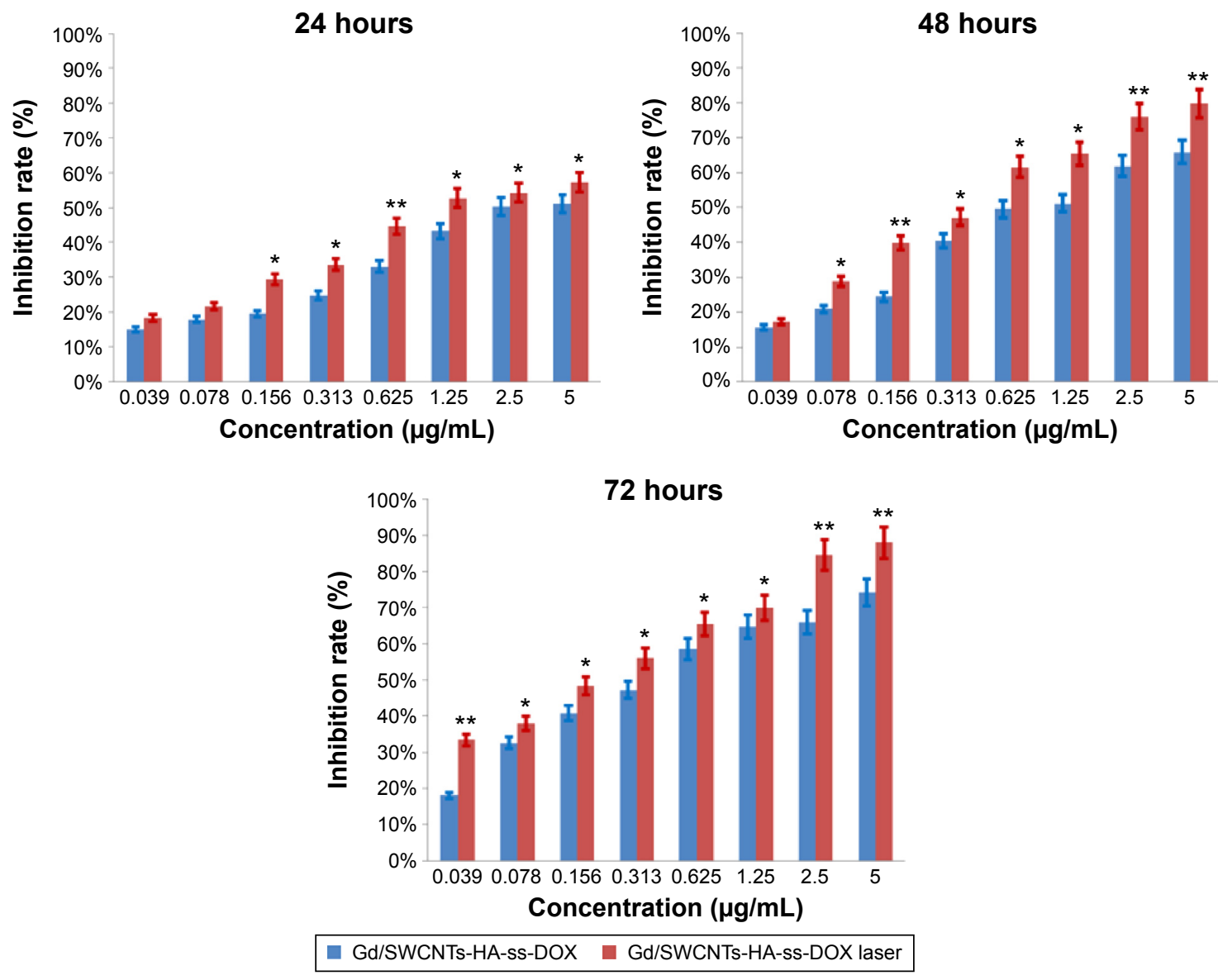

Figure 7 Cell inhibition with or without NIR laser irradiation on MCF-7 cells (mean \pm standard deviation, $\mathrm{n}=6$ ).

Notes: $* P<0.05$; $* * P<0.01$.

Abbreviations: DOX, doxorubicin; Gd, gadolinium; HA, hyaluronic acid; MCF-7, Michigan Cancer Foundation-7; NIR, near-infrared; SWCNTs, single-walled carbon nanotubes. 
or equivalently higher mortality of the cells. Interestingly, the antitumor activity of Gd/SWCNTs-HA-ss-DOX $\left(\mathrm{IC}_{50}=3.37,1.0\right.$, and $0.43 \mu \mathrm{g} \mathrm{DOX}$ equiv/mL at 24,48 , and 72 hours, respectively) was higher than that of free DOX $\left(\mathrm{IC}_{50}=4.51,1.83\right.$, and $0.89 \mu \mathrm{g}$ DOX equiv $/ \mathrm{mL}$ at 24,48 , and 72 hours, respectively). In theory, DOX is a small molecule and it could be quickly transported into the nuclei by passive diffusion, leading to strong cytotoxicity. However, based on the drug release and cellular uptake results, HA-modified nanotubes could be internalized by high-efficacy endocytosis and then rapidly release the disulfide bond-conjugated drugs in response to GSH, and correspondingly exhibit enhanced cytotoxicity.

Moreover, it should be noted that SWCNTs-HA-ss-DOX and $\mathrm{Gd} / \mathrm{SWCNTs-HA}$-ss-DOX had low $\mathrm{IC}_{50}$ values of 0.99 and $1.0 \mu \mathrm{g}$ DOX equiv $/ \mathrm{mL}$ at 48 hours, respectively, which were significantly lower than that obtained with the redoxinsensitive counterparts under otherwise the same conditions $\left(\mathrm{IC}_{50}=198.33 \mathrm{DOX}\right.$ equiv $\left./ \mathrm{mL}\right)$. This result further indicated that the cytotoxicity was induced by the released DOX from the conjugate, which was internalized into the cell through uptake via intracellular enzymolysis. Besides, there was nearly no difference between SWCNTs-HA-ss-DOX with and without $\mathrm{Gd}^{3+}$-ion loaded. It suggested that after loading on the nanotubes, $\mathrm{GdCl}_{3}$ did not show enhanced toxicity.

Finally, the synergetic effect of DOX and SWCNTs-HA (a photothermal agent) was accessed under irradiation by NIR laser. Tumor cell killing potency was enhanced when Gd/SWCNTs-HA-ss-DOX were combined with NIR irradiation. For example, there was $\sim 80 \%$ cell growth inhibition induced by NIR at the equivalent DOX concentration of 5 $\mu \mathrm{g} / \mathrm{mL}$ at 48 hours, in comparison with $\sim 62 \%$ cell growth inhibition without NIR irradiation. It demonstrated that $\mathrm{Gd} /$ SWCNTs-HA-ss-DOX under laser irradiation could selectively carry heat and drug to cancer cells and significantly enhance the therapeutic efficacy of chemo-photothermal treatment. The results were in accordance with the previous works of nanocomposites based on SWCNTs or graphene oxide nanosheets, which promoted the diffusion of drug and provided the photothermal treatment. ${ }^{29}$

\section{Cell cycle and apoptosis assays}

As shown Figure 8A, analysis of cell cycle indicated that the Gd/SWCNTs-HA-ss-DOX could effectively arrest MCF-7 cells in the S phase after 24 hours incubation. Compared with SWCNTs-HA-ss-DOX, Gd/SWCNTs-HAss-DOX had a relatively higher arrest proportion of cells in the $\mathrm{S}$ phase, but there were no significant differences
$\mathbf{A}^{4} \mathrm{a}$

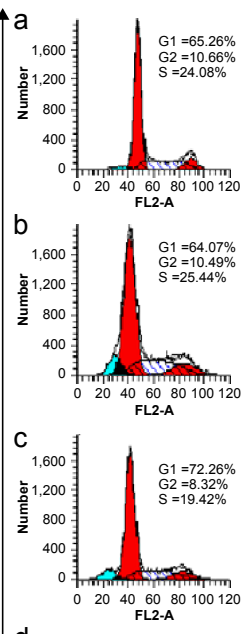

d

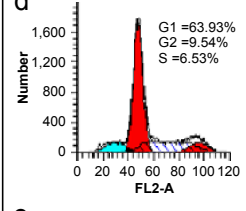

e

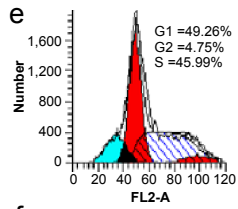

f

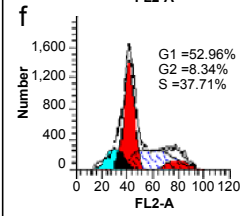

g

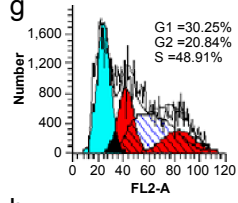

h

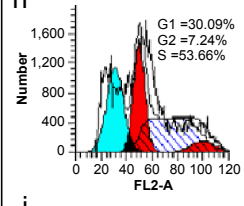

i

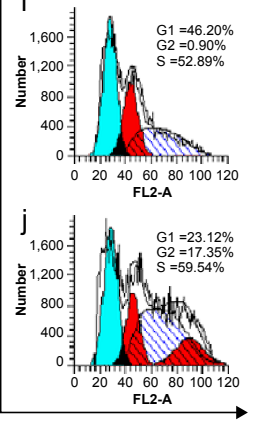

B

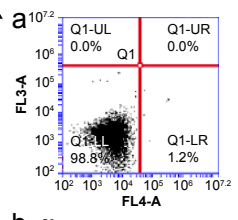

$b_{10}$
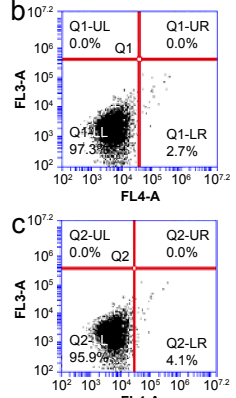

d $100^{2}$

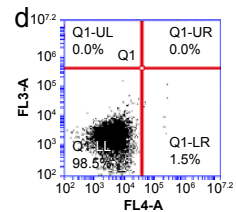

$\mathrm{e}^{10^{72}} \overline{\text { Q1-UL }} \mid$ Q1-UR

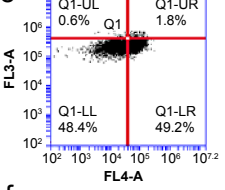

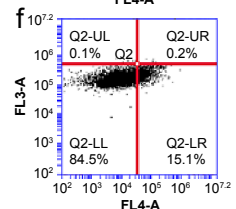

$\mathrm{g}^{10^{2 x}}$

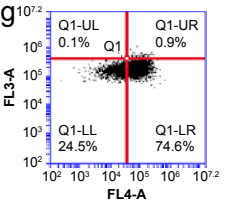

$\mathrm{h}_{10}$

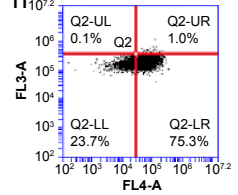

$\mathrm{i}_{10}$
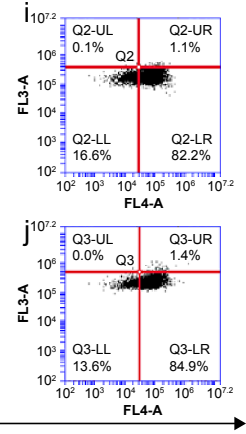

Figure $\mathbf{8}$ Cell apoptosis without $(\mathbf{B g}, \mathbf{B h})$ or with laser irradiation $(\mathbf{B i}, \mathbf{B j})$ and cell cycle distribution without $(\mathbf{A g}, \mathbf{A})$ or with laser irradiation $(\mathbf{A i}, \mathbf{A j})$ after treated with different DOX formulations in MCF-7 cells. Cell cycle distribution and cell apoptosis for blank cells (Aa, Ba), SWCNTs-HA without (Ab, Bb) or with (Ac, Bc) laser irradiation and $\mathrm{Gd}(\mathbf{A d}, \mathbf{B d})$.

Abbreviations: DOX, doxorubicin; Gd, gadolinium; HA, hyaluronic acid; MCF-7, Michigan Cancer Foundation-7; SWCNTs, single-walled carbon nanotubes. 
Table I The representative flow cytometry data for tumor-specific induction of apoptosis

\begin{tabular}{|c|c|c|c|c|c|c|c|c|c|c|}
\hline & $\mathbf{a}$ & b & c & d & e & f & $\mathbf{g}$ & $\mathbf{h}$ & i & j \\
\hline Early stage apoptotic rate (\%) & 1.2 & 2.7 & 4.1 & 1.5 & 49.2 & I5.I & 74.6 & 75.3 & 82.2 & 84.9 \\
\hline Late stage apoptotic rate (\%) & 0 & 0 & 0 & 0 & 1.8 & 0.2 & 0.9 & 1.0 & I.I & 1.4 \\
\hline Total apoptotic rate (\%) & 1.2 & 2.7 & 4.1 & 1.5 & 51.0 & 15.3 & 75.5 & 76.3 & 83.3 & 86.3 \\
\hline
\end{tabular}

Notes: Cell apoptosis groups: a) blank, b) SWCNTs-HA, c) SWCNTs-HA/laser, d) Gd, e) DOX, f) Gd/SWCNTs-HA-DOX, g) SWCNT-HA-ss-DOX, h) Gd/SWCNTs-HAss-DOX, i) SWCNT-HA-ss-DOX/laser, j) Gd/SWCNTs-HA-ss-DOX/laser.

Abbreviations: DOX, doxorubicin; Gd, gadolinium; HA, hyaluronic acid; SWCNTs, single-walled carbon nanotubes; SWCNTs-HA, HA-modified SWCNTs.

between these groups. This might be due to the slight influence of $\mathrm{GdCl}_{3}$. By contrast, the differences between Gd/SWCNTs-HA-ss-DOX and Gd/SWCNTs-HA-DOX were obvious. The lower arrest proportion of cells in the S phase for Gd/SWCNTs-HA-DOX suggested that activation of apoptotic signals to induce tumor cell death might be induced by the rapid drug release at the tumor site from the redox-sensitive nanotubes..$^{30}$ On the other hand, $\mathrm{Gd} /$ SWCNTs-HA-ss-DOX and SWCNTs-HA-ss-DOX with $808 \mathrm{~nm}$ NIR laser irradiation also demonstrated enhanced arrest in the S phase, owing to the effect of photothermal treatment.

The representative flow cytometry images for tumorspecific induction of apoptosis are shown in Figure 8B and Table 1. Treatment of Gd/SWCNTs-HA-ss-DOX with 808 $\mathrm{nm}$ laser irradiation displayed strong antitumor efficacy on MCF-7 cancer cells, due to the combination of redox-sensitive and photothermal properties of formulation. ${ }^{31}$ The blank cells exhibited a good growth state (Figure $8 \mathrm{~B}$ [a]), while early stage apoptotic cells were observed after treatment with SWCNTs-HA (2.7\%, Figure 8B [b]), and after laser irradiation, the amount of early stage apoptotic cells increased to $4.1 \%$ (Figure 8B [c]). Significantly, early stage apoptotic cells and late stage apoptotic cells were both observed after treatment with all formulations (Figure 8B [d]-[f]). Compared with free $\operatorname{DOX}(51 \%$, Figure $8 \mathrm{~B}[\mathrm{e}])$, there were more apoptotic cells for SWCNT-HA-ss-DOX (75.5\%, Figure 8B [g]) and Gd/SWCNTs-HA-ss-DOX (76.3\%, Figure 8B [h]), suggesting that death of tumor cells was caused by apoptotic pathway after redox-sensitive nanotubes treatment, which was further proved by the apoptotic cells in nonsensitive $\mathrm{Gd} /$ SWCNTs-HA-DOX (15.3\%, Figure 8B [f]).

Moreover, we also confirmed the photothermal killing effect of SWCNTs-HA-ss-DOX and Gd/SWCNTs-HA-ssDOX. As can be seen in Figure 8B (i, j), upon laser irradiation, the amount of apoptotic cells increased to $83.3 \%$ and $86.3 \%$, respectively, indicating that SWCNTs-HA-ss-DOX could be an outstanding photothermal agent and the redoxsensitive properties associating with laser irradiation induced cancer cells to die through cell apoptosis manner. ${ }^{32}$

\section{In vivo $M R I$}

MRI is a powerful technique in clinical diagnostics such as tumor detection and vascular imaging. New Gd-based complexes in our work have been designed to obtain highresolution MRI. As shown in Figure 9, Gd/SWCNTs-HAss-DOX showed the $\mathrm{T}_{1}$-weighted positive contrast-enhanced MRI capability for the tumor tissues after intravenous administration. The tumor vasculature in the periphery showed a significant contrast enhancement at the initial stage, displaying the accumulation of $\mathrm{Gd} / \mathrm{SWCNTs-HA}$-ssDOX around the tumor tissue by the enhanced permeability and retention effect. ${ }^{33}$ Importantly, the tumor interior of $\mathrm{Gd} /$ SWCNTs-HA-ss-DOX exhibited much stronger $\mathrm{T}_{1}$ contrast enhancements gradually during the time course than $\mathrm{Gd} /$ SWCNTs-COOH, indicating the HA-mediated tumor targeting ability. It was also found that although there was an immediate enhancement in signal intensity for Gd-DTPA group at first 30 minutes, it decreased quickly to the normal level. ${ }^{34}$ The results of MRI measurement suggested that $\mathrm{Gd} /$ SWCNTs-HA-ss-DOX had potential as MRI CA capable of systemic tumor targeting.

\section{In vivo imaging analysis}

Based on the in vivo MRI results, the targeted tumor accumulation of HA-modified nanotube was further confirmed using a noninvasive NIR optical imaging technique. The tumor-bearing mice were intravenously injected with IR-783, IR-783/SWCNTs-COOH, and IR-783/SWCNTs-HA-ssDOX, respectively. As shown in Figure 10, for all the samples, considerable fluorescence signals were detected in the whole bodies of the mice at the early time points ( $0.5-1$ hours), resulting from circulation in the bloodstream, and then gradually decreased as a function of time. For the IR-783 group, strong signals were detected in kidney and liver following by the weak signals in tumor during 6 and 8 hours, indicating that IR-783 could be cleared rapidly by kidney during the blood circulation without significant tumor accumulation. ${ }^{35,36}$

On the other hand, the SWCNTs-HA-ss-DOX and SWCNTs-COOH exhibited a strong fluorescence signal for up to 8 hours, which was possible due to the prolonged 


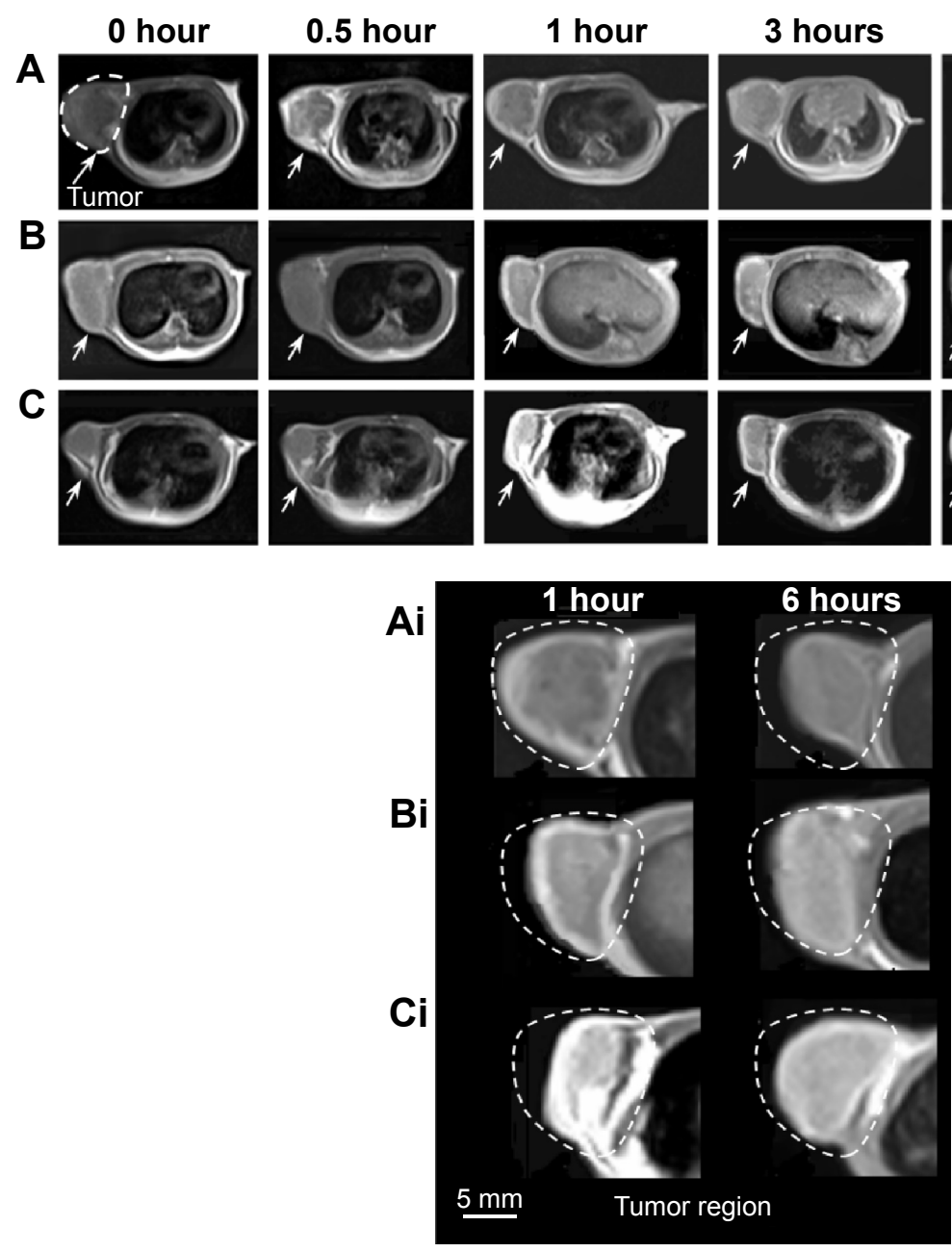

Figure 9 In vivo T,-weighted MRI images of (A) Gd-DTPA, (B) Gd/SWCNTs-COOH, and (C) Gd/SWCNTs-HA-ss-DOX. Notes: Arrows show the location of the tumor. Ai, Bi, Ci refer to the magnification of $\mathbf{A}, \mathbf{B}, \mathbf{C}$ at I hour and 6 hours.

Abbreviations: DOX, doxorubicin; Gd, gadolinium; HA, hyaluronic acid; MRI, magnetic resonance imaging; SWCNTs, single-walled carbon nanotubes.

circulation characteristics of the nanotubes. Moreover, the fluorescent signals in IR-783/SWCNTs-HA-ss-DOX group were much stronger than IR-783/SWCNTs-COOH group in tumor site at the same time. Since CD44 in tumor cells is one of the specific receptors of HA, the high distribution of IR-783 in tumor seemed reasonable and was in accordance with the previous report. ${ }^{37}$ This high tumor targetability of SWCNTs-HA-ss-DOX might be due to a combination of an enhanced permeability and retention effect and receptormediated uptake of nanotubes. Overall, these results indicate that SWCNTs-HA-ss-DOX could accumulate into tumor and retain for a long time, while the nanotubes might be stable during the blood circulation, leading to high antitumor efficacy and good MRI ability.

\section{In vivo antitumor efficacy studies}

The in vivo therapeutic effect of Gd/SWCNTs-HA-ss-DOX on tumor was evaluated using S-180 tumor-bearing mice. Mice were divided into nine groups and treated with the protocols summarized in 'In vivo antitumor efficacy studies' in the Materials and methods section. The changes in relative tumor volume $\left(\mathrm{V} / \mathrm{V}_{0}\right)$ as a function of time are plotted in Figure 11A.

After treatment for 10 days, the relative tumor volume $\left(\mathrm{V} / \mathrm{V}_{0}\right)$ of the saline group and $\mathrm{Gd} / \mathrm{SWCNTs-HA}-\mathrm{DOX}$ without $808 \mathrm{~nm}$ laser irradiation was $560 \% \pm 31 \%$ and $430 \% \pm 37 \%$, respectively, suggesting that the two groups would not affect the tumor growth. In comparison, DOX only showed moderate antitumor efficacy, resulting in a relative tumor volume of $265 \% \pm 32 \%$ at day 10 . By contrast, the tumor-bearing mice treated with SWCNTs-HA-ss-DOX and Gd/SWCNTsHA-ss-DOX exhibited a significantly stronger response than that treated with free drug DOX and nonsensitive nanotubes, leading to a relative tumor volume of $131 \% \pm 33 \%$ and $142 \% \pm 32 \%$ at day 10 , respectively. Moreover, final tumor weight was measured and inhibition ratios were calculated. The results also demonstrated that SWCNTs-HA-ss-DOX 

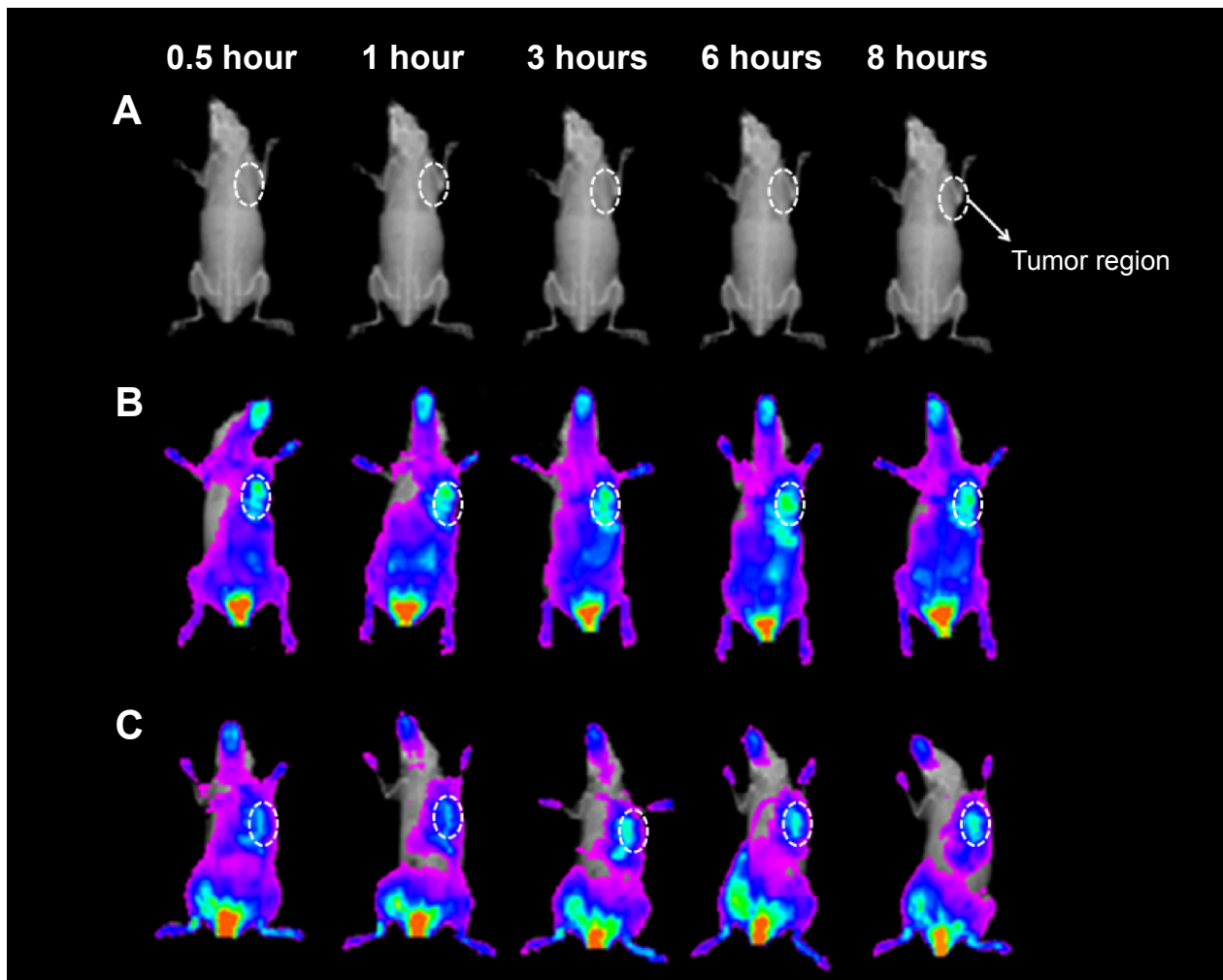

D
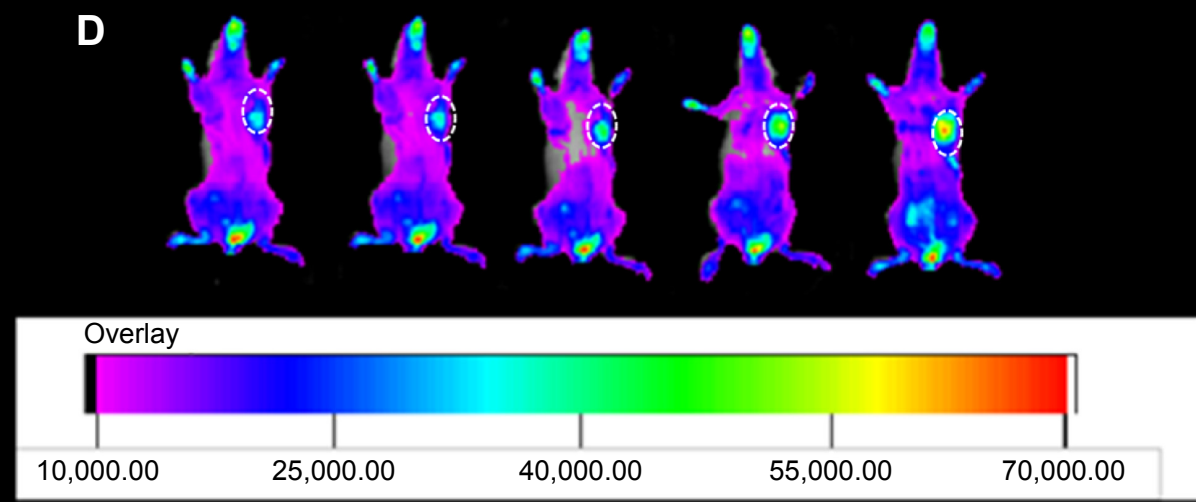

Figure 10 In vivo imaging of tumor-bearing mice administered with (A) saline control, (B) IR-783, (C) IR-783/SWCNTs-COOH, and (D) IR-783/SWCNTs-HA-ss-DOX. Abbreviations: DOX, doxorubicin; HA, hyaluronic acid; SWCNTs, single-walled carbon nanotubes.

and Gd/SWCNTs-HA-ss-DOX inhibited tumor growth most efficiently (70.14\% and $71.27 \%$ ), followed by DOX solution (40.74\%), which were consistent with the results from tumor volume measurements. This can be explained by the redox selective release of DOX from redox-sensitive nanotubes upon internalization into tumor cells. On the other hand, free DOX could not achieve obvious antitumor effect due to its short half-life in blood ${ }^{37-39}$ and weak antitumor efficiency of $\mathrm{Gd} / \mathrm{SWCNTs-HA-DOX}$ could be attributed to nearly no DOX release from the redox-insensitive nanotubes.

After laser irradiation, the tumor sizes in all formulations were decreased compared with the control groups without irradiation as above. But there was no significant difference between the saline group and saline + laser group, or DOX and DOX + laser group, demonstrating that laser irradiation did cause tumor growth inhibition through SWCNTs. This synergistic enhanced therapeutic efficacy originated from the high DOX and SWCNTs-HA accumulation in tumor tissue to achieve targeting chemophotothermal therapy.

Finally, typical histological images of tumor tissue are shown in Figure 11B. Tumor cells treated with saline showed rapid proliferation and close cell-to-cell arrangement in a good condition. But it could be clearly observed in DOX 

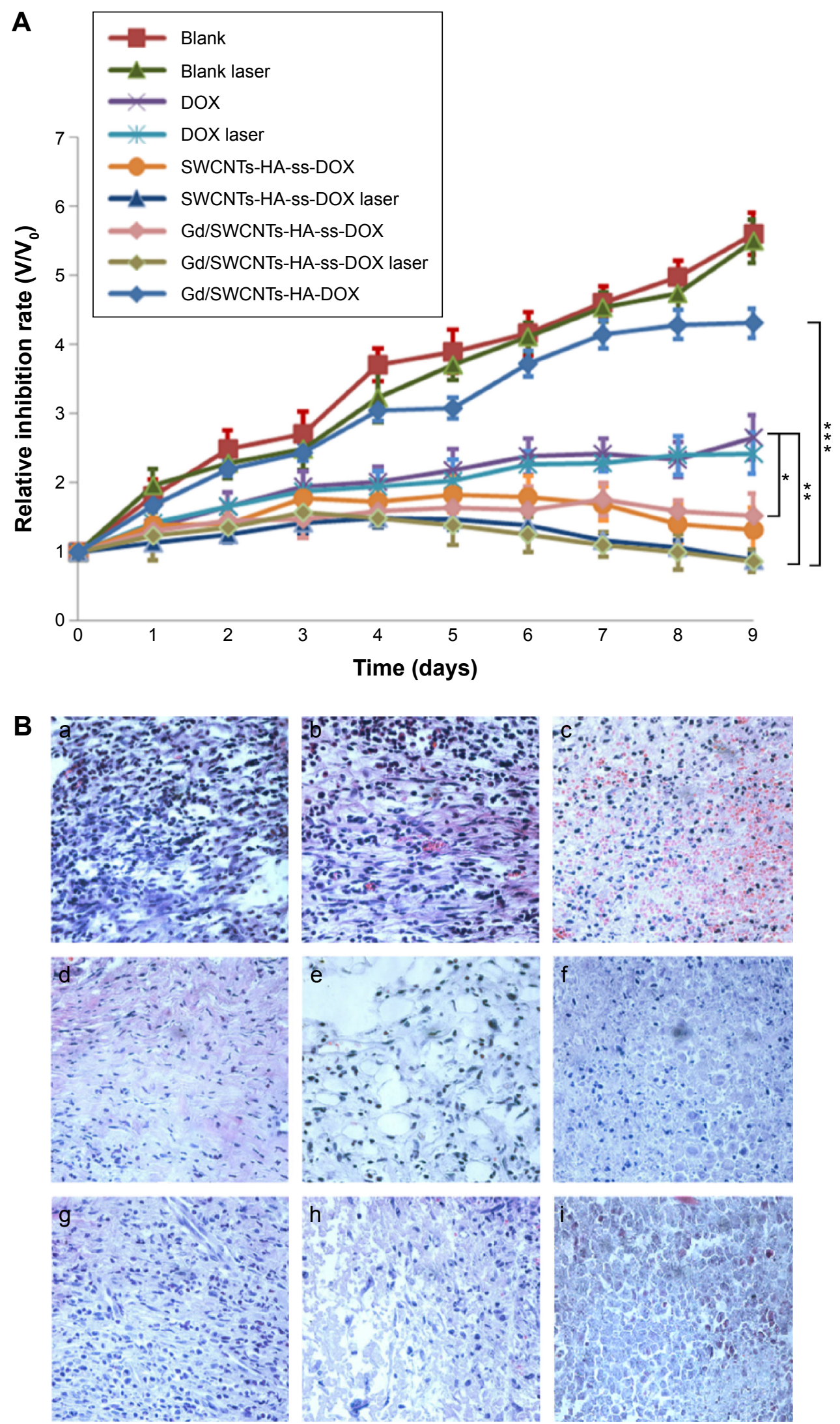

Figure II In vivo antitumor efficacy assay.

Notes: (A) Tumor volumes of tumor-bearing mice as a function of time. Data were given as mean $(n=6)$. (B) Tissue sections of tumor excised from the mice 7 days after administration of a) saline, b) saline + laser, c) DOX, d) DOX + laser, e) SWCNTs-HA-ss-DOX, f) SWCNTs-HA-ss-DOX + laser, g) Gd/SWCNTs-HA-ss-DOX, h) Gd/ SWCNTs-HA-ss-DOX + laser, and i) Gd/SWCNTs-HA-DOX. *P<0.05; **P<0.01; *** $P<0.001$.

Abbreviations: DOX, doxorubicin; Gd, gadolinium; HA, hyaluronic acid; SWCNTs, single-walled carbon nanotubes. 
group that tumor cells began to shrink and there was clear gap emerging among cells, indicating that DOX was toxic to tumor cells and the state of cell growth had begun to change severely. ${ }^{32,38}$ Furthermore, in SWCNTs-HA-ss-DOX, Gd/ SWCNTs-HA-ss-DOX, SWCNTs-HA-ss-DOX + laser group, and Gd/SWCNTs-HA-ss-DOX + laser group, typical symptoms including obvious necrosis, karyotheca dissolving, and nucleus disappearing all appeared. Especially in $\mathrm{Gd} /$ SWCNTs-HA-ss-DOX + laser group, there were almost no intact tumor cells, displaying that Gd/SWCNTs-HA-ss-DOX with $808 \mathrm{~nm}$ laser irradiation could achieve a perfect synergistic antitumor effect in vivo. In addition, compared with $\mathrm{Gd} /$ SWCNTs-HA-ss-DOX, there were just few adverse changes in $\mathrm{Gd} / \mathrm{SWCNTs-HA-DOX}$ due to low release of DOX without redox-sensitive disulfide bond conjugation.

\section{Conclusion}

In this work, a multifunctional tumor targeting SWCNTsHA-ss-DOX with redox sensitivity was successfully synthesized for effective co-delivery of DOX and photothermal agent SWCNTs. MRI CA Gd was further loaded into this conjugate for cancer diagnosis. This system was stable at normal physiologic condition but readily cleaved to release drugs under reducing environment. Most importantly, Gd/SWCNTs-HA-ss-DOX exhibited excellent chemo-photothermal anticancer therapeutic efficacy, tumor targeting property, redox-responsive releasing function, and MRI ability. Therefore, this system has a great potential for targeted cancer theranostic application.

\section{Acknowledgments}

This work was supported by grants from the National Natural Science Foundation of China (81202485 and 81273451).

\section{Disclosure}

These authors report no conflicts of interest in this work.

\section{References}

1. Xie J, Lee S, Chen X. Nanoparticle-based theranostic agents. Adv Drug Deliv Rev. 2010;62:1064-1079.

2. Yoon HY, Saravanakumar G, Heo R, et al. Hydrotropic magnetic micelles for combined magnetic resonance imaging and cancer therapy. J Control Release. 2012;160:692-698.

3. Peer D, Karp JM, Hong S, Farokhzad OC, Margalit R, Langer R. Nanocarriers as an emerging platform for cancer therapy. Nat Nanotechnol. 2007;2:751-760.

4. Kim K, Kim JH, Park H, et al. Tumor-homing multifunctional nanoparticles for cancer theragnosis: simultaneous diagnosis, drug delivery, and therapeutic monitoring. J Control Release. 2010;146:219-227.

5. Li Z, Wang Y, Pei Y, et al. Curcumin encapsulated in the complex of lysozyme/carboxymethylcellulose and implications for the antioxidant activity of curcumin. Food Res Int. 2011;75:98-105.
6. Liu Y, Zhang N. Gadolinium loaded nanoparticles in theranostic magnetic resonance imaging. Biomaterials. 2012;33:5363-5375.

7. Sitharaman B, Kissell KR, Hartman KB, et al. Superparamagnetic gadonanotubes are high-performance MRI contrast agents. Chem Commun (Camb). 2005;31:3915-3917.

8. Tran LA, Krishnamurthy R, Muthupillai R, et al. Gadonanotubes as magnetic nanolabels for stem cell detection. Biomaterials. 2010;31: 9482-9491.

9. Ji Z, Lin G, Lu Q, et al. Targeted therapy of SMMC-7721 liver cancer in vitro and in vivo with carbon nanotubes based drug delivery system. J Colloid Interface Sci. 2012;365:143-149.

10. Liu X, Tao H, Yang K, Zhang S, Lee ST, Liu Z. Optimization of surface chemistry on single-walled carbon nanotubes for in vivo photothermal ablation of tumors. Biomaterials. 2011;32:144-151.

11. Meng L, Zhang X, Lu Q, Fei Z, Dyson PJ. Single walled carbon nanotubes as drug delivery vehicles: targeting doxorubicin to tumors. Biomaterials. 2012;33:1689-1698.

12. Choi KY, Chung H, Min KH, et al. Self-assembled hyaluronic acid nanoparticles for active tumor targeting. Biomaterials. 2010;31:106-114.

13. Lee H, Lee K, Park TG. Hyaluronic acid-paclitaxel conjugate micelles: synthesis, characterization, and antitumor activity. Bioconjug Chem. 2008;19:1319-1325.

14. Lim EK, Kim HO, Jang E, et al. Hyaluronan-modified magnetic nanoclusters for detection of CD44-overexpressing breast cancer by MR imaging. Biomaterials. 2011;32:7941-7950.

15. Kaminskas LM, Kelly BD, McLeod VM, et al. Characterisation and tumour targeting of PEGylated polylysine dendrimers bearing doxorubicin via a pH labile linker. J Control Release. 2011;152:241-248.

16. Agarwal A, Gupta U, Asthana A, Jain NK. Dextran conjugated dendritic nanoconstructs as potential vectors for anti-cancer agent. Biomaterials. 2009;30:3588-3596.

17. Li J, Huo M, Wang J, et al. Redox-sensitive micelles self-assembled from amphiphilic hyaluronic acid-deoxycholic acid conjugates for targeted intracellular delivery of paclitaxel. Biomaterials. 2012;33: 2310-2320.

18. Meng F, Hennink WE, Zhong Z. Reduction-sensitive polymers and bioconjugates for biomedical applications. Biomaterials. 2009;30: 2180-2198.

19. Cheng R, Feng F, Meng F, Deng C, Feijen J, Zhong Z. Glutathioneresponsive nano-vehicles as a promising platform for targeted intracellular drug and gene delivery. J Control Release. 2011;152:2-12.

20. Cheng R, Meng F, Deng C, Klok HA, Zhong Z. Dual and multi-stimuli responsive polymeric nanoparticles for programmed site-specific drug delivery. Biomaterials. 2013;34:3647-3657.

21. Hou L, Feng Q, Wang Y, et al. Multifunctional hyaluronic acid modified graphene oxide loaded with mitoxantrone for overcoming drug resistance in cancer. Nanotechnology. 2016;27:051701.

22. Hou L, Zhang H, Wang Y, Wang L, Yang X, Zhang Z. Hyaluronic acid-functionalized single-walled carbon nanotubes as tumor-targeting MRI contrast agent. Int J Nanomedicine. 2015;10:4507-4520.

23. Sitharaman B, Wilson LJ. Gadonanotubes as new high-performance MRI contrast agents. Int J Nanomedicine. 2006;1:291-295.

24. Kuppusamy $\mathrm{P}, \mathrm{Li} \mathrm{H}$, Ilangovan $\mathrm{G}$, et al. Noninvasive imaging of tumor redox status and its modification by tissue. Cancer Res. 2002;62: 307-312.

25. Li J, Huo M, Wang J, et al. Redox-sensitive micelles self-assembled from amphiphilic hyaluronic acid-deoxycholic acid conjugates for targeted intracellular delivery of paclitaxel. Biomaterials. 2012;33: 2310-2320.

26. Saito G, Swanson JA, Lee K-D. Drug delivery strategy utilizing conjugation via reversible disulfide linkages: role and site of cellular reducing activities. Adv Drug Deliv Rev. 2003;55:199-215.

27. Cui C, Xue YN, Wu M, et al. Cellular uptake, intracellular trafficking, and antitumor efficacy of doxorubicin-loaded reduction-sensitive micelles. Biomaterials. 2013;34:3858-3869.

28. Wang L, Shi J, Jia X, et al. NIR-/pH-Responsive drug delivery of functionalized single-walled carbon nanotubes for potential application in cancer chemo-photothermal therapy. Pharm Res. 2013;30:2757-2771. 
29. Yao HJ, Zhang YG, Sun L, Liu Y. The effect of hyaluronic acid functionalized carbon nanotubes loaded with salinomycin on gastric cancer stem cells. Biomaterials. 2014;35:9208-9223.

30. Hou L, Feng Q, Wang Y, et al. Multifunctional nanosheets based on hyaluronic acid modified graphene oxide for tumor-targeting chemophotothermal therapy. J Nanopart Res. 2015;17:1-17.

31. Ji BS, He L, Liu GQ. Reversal of p-glycoprotein-mediated multidrug resistance by CJX1, an amlodipine derivative, in doxorubicin-resistant human myelogenous leukemia (K562/DOX) cells. Life Sci. 2005;77: 2221-2232.

32. Wang J, Yang G, Guo X, Tang Z, Zhong Z, Zhou S. Redox-responsive polyanhydride micelles for cancer therapy. Biomaterials. 2014;35: 3080-3090.

33. Zhang H, Hou L, Jiao X, Ji Y, Zhu X, Zhang Z. Transferrin-mediated fullerenes nanoparticles as $\mathrm{Fe}(2+)$-dependent drug vehicles for synergistic anti-tumor efficacy. Biomaterials. 2015;37:353-366.

34. Chen Y, Yin Q, Ji X, et al. Manganese oxide-based multifunctionalized mesoporous silica nanoparticles for $\mathrm{pH}$-responsive MRI, ultrasonography and circumvention of MDR in cancer cells. Biomaterials. 2012;33:7126-7137.
35. Liu Y, Chen Z, Liu C, Yu D, Lu Z, Zhang N. Gadolinium-loaded polymeric nanoparticles modified with Anti-VEGF as multifunctional MRI contrast agents for the diagnosis of liver cancer. Biomaterials. 2011; 32:5167-5176.

36. Yoon HY, Koo H, Choi KY, et al. Tumor-targeting hyaluronic acid nanoparticles for photodynamic imaging and therapy. Biomaterials. 2012;33:3980-3989.

37. Qiu L, Qiao M, Chen Q, et al. Enhanced effect of $\mathrm{pH}$-sensitive mixed copolymer micelles for overcoming multidrug resistance of doxorubicin. Biomaterials. 2014;35:9877-9887.

38. Choi KY, Min KH, Na JH, et al. Self-assembled hyaluronic acid nanoparticles as a potential drug carrier for cancer therapy: synthesis, characterization, and in vivo biodistribution. J Mater Chem. 2009;19:4102.

39. Hou L, Yao J, Zhou J, Zhang Q. Pharmacokinetics of a paclitaxelloaded low molecular weight heparin-all-trans-retinoid acid conjugate ternary nanoparticulate drug delivery system. Biomaterials. 2012;33: $5431-5440$
International Journal of Nanomedicine

\section{Publish your work in this journal}

The International Journal of Nanomedicine is an international, peerreviewed journal focusing on the application of nanotechnology in diagnostics, therapeutics, and drug delivery systems throughout the biomedical field. This journal is indexed on PubMed Central, MedLine, CAS, SciSearch ${ }^{\circledR}$, Current Contents ${ }^{\circledR} /$ Clinical Medicine,

\section{Dovepress}

Journal Citation Reports/Science Edition, EMBase, Scopus and the Elsevier Bibliographic databases. The manuscript management system is completely online and includes a very quick and fair peer-review system, which is all easy to use. Visit http://www.dovepress.com/ testimonials.php to read real quotes from published authors. 